\title{
Checklist of the Cnidaria Medusozoa of Brazil
}

\author{
Alvaro E. Migotto, Antonio C. Marques, André C. Morandini, Fábio L. da Silveira
}

Biota Neotropica Volume 2(1) - http://www.biotaneotropica.org.br/v2n1/pt/fullpaper?bn01102012002+en

\author{
Date Received: 02/25/2002 \\ Revised: 04/15/2002 \\ Accepted:04/19/2002
}

Centro de Biologia Marinha, Universidade de São Paulo, Caixa Postal 83, 11600-970, São Sebastião, SP, Brazil. Departamento de Zoologia, Instituto de Biociências, Universidade de São Paulo, Caixa Postal 11461, 05422-970, São Paulo, SP, Brazil.

e-mails: aemigott@usp.br, marques@ib.usp.br, acmorand@usp.br, fldsilve@usp.br Autor para correspondência: Alvaro E. Migotto aemigott@usp.br

\begin{abstract}
Checklist of the Cnidaria Medusozoa from Brazil

Literature records were reviewed to compile a list of species of the marine taxa of Cnidaria Medusozoa recorded for the Brazilian coast. The total number of species of medusozoans so far recorded for Brazil is 373: 347 Hydrozoa, 3 Cubozoa: 23 Scyphozoa.
\end{abstract}

Keywords: Hydrozoa, Scyphozoa, Cubozoa, fauna, South Atlantic, Brazil, polyp, medusa

\section{Resumo}

Lista dos Cnidaria Medusozoa do Brasil

Uma lista dos Cnidaria Medusozoa marinhos do Brasil foi composta a partir de registros de ocorrência disponíveis na literatura. Até o momento, há um total de 373 espécies registradas para o Brazil: 347 de Hydrozoa, 3 de Cubozoa e 23 de Scyphozoa.

Palavras-chave: hidrozoários, cifozoários, cubozoários, fauna, Atlântico Sul, Brasil, pólipo, medusa 
The tradition of systematics in Brazil is still evolving, with interest having increased markedly in recent years. Nevertheless, there are several higher groups, including phyla, without a single species record along the entire Brazilian coast (see Migotto and Tiago, 1999, for examples from São Paulo state, SE Brazil). This is due to the small number of taxonomists, who have worked on relatively few groups, in the country. Moreover, there are few natural history museums in Brazil and conservation of their collections has suffered due to limited personnel and modest budgets. Brazilian biodiversity is certainly underestimated given the large size of the country, and the range and complexity of its ecosystems. The coastline, for example, extends a distance of some $8,000 \mathrm{~km}$ and encompasses a range of physiographic provinces from the subtropical South to the equatorial North.

A few isolated programs have been undertaken thus far to address marine biodiversity. One recently established program, called BIOTA, aims to survey the organisms of São Paulo state (SE Brazil). One of the initial steps of any biodiversity program is a survey of previously published data. The purpose of this study is to summarize, in a tabular checklist, available knowledge from the literature on medusozoans of the Brazilian coast.

A list of marine taxa of Cnidaria Medusozoa from Brazil, compiled from literature records, is given below. As a general rule, we followed major treatises on medusozoans (e.g. Mayer 1910, Kramp 1961, Calder 1988, 1991, 1997, Mianzan \& Cornelius 1999, Pugh 1999) regarding the taxonomic status and classification of species. Whenever a record was considered doubtful in the original work, we preserved the information using a question mark in front of the author citation in the references column of the table. We also decided to include nominal species of uncertain validity so as not to lose potentially useful information. Broad distributional ranges, for instance those provided by plankton guides, were considered as a continuous range of occurrence from north to south of the species. Vague geographical information was not detailed in the table (e.g. "Brazil" is treated as "no specific record for Brazil"). Whenever pertinent, information regarding the occurrence of medusa (M), or polyp $(\mathrm{P})$, or both, in Brazilian waters was included. Codes used for the states, from south to north of the country, are: RS (Rio Grande do Sul); SC (Santa Catarina); PR (Paraná); SP (São Paulo); RJ (Rio de Janeiro); ES (Espírito Santo); BA (Bahia); SE (Sergipe); AL (Alagoas); PE (Pernambuco) PB (Paraíba); RN (Rio Grande do Norte); CE (Ceará); PI (Piauí); MA (Maranhão); PA (Pará); AP (Amapá). Oceanic islands were also individually considered and abbreviated as AR (Atol da Rocas); FN (Fernando de Noronha) and TRI (Trindade Island). The regions adopted in the study are as defined geopolitically; these are abbreviated as S (South, includes the states RS, SC, PR); SE (Southeast, includes SP, RJ, ES); NE (Northeast, includes BA, SE, AL, PE, PB, RN, CE, PI, MA, plus the islands $\mathrm{AR}$ and $\mathrm{FN}$ ) and $\mathrm{N}$ (North, includes PA, AM, AP).
All classes of Medusozoa are represented along the coast of Brazil. The total number of species of medusozoans so far recorded for Brazil is 373 (Hydrozoa: 347; Cubozoa: 3; Scyphozoa: 23), arranged in 194 genera and 83 families (Table 1).

\section{Acknowledgements}

The authors thank Clóvis B. Castro and Débora O. Pires (MN-UFRJ), Erika Schlenz and Otto M.P. Oliveira (IBUSP) for the help in the elaboration of the data bank. We also thank an anonymous reviewer whose comments improved the manuscript. The study received financial support from the State of São Paulo Research Foundation (FAPESP) within the BIOTA/FAPESP - The Biodiversity Virtual Institute Program (www.biota.org.br) (proc. $\mathrm{n}^{\circ}$ 1998/07090-3) and other projects (proc. no 1996/10544-0, 1998/11072-0, 1999/05374-7, 1999/12433-0, 2001/02626-7). A.C. Marques and A.E. Migotto have financial support from CNPq.

\section{References}

ALCÂNTARA, A.V., ROCHA, C.E.F. \& SANTOS, M.A. 1979. Caracterização hidrológica e biológica do estuário do Rio Sergipe. Sergipe, Unigráfica (UFSE).

ALLMAN, G.J. 1883. Report on the Hydroida dredged by H.M.S. Challenger during the years 1873-76. Rep. Sci. Res. Challenger Exp. (Zool. Ser.) 7(20): 1-55.

ALLMAN, G.J. 1888. Report on the Hydroida dredged by H.M.S. Challenger during the years 1873-76. Part II. The Tubularinae, Corymorphinae, Campanularinae, Sertularinae and Thalamophora. Rep. Sci. Res. Challenger Exp. (Zool. Ser.) 23: 1-90.

ALVARIñO, A. 1968. Los quetognatos, sifonoforos y medusas en la región del Atlantico ecuatorial bajo la influencia del Amazonas. An. Inst. Biol., Univ. Auton. Méx., Ser. Mar Limnol 1:41-76.

ALVARIñO, A. 1971. Siphonophores of the Pacific with a review of the world distribution. Bull. Scripps Inst. Oceanogr. 16: 1-432.

ALVARIñO, A. 1981. Siphonophorae. In Atlas del zooplancton del Atlántico sudoccidental y metodos de trabajo con el zooplancton marino (D. Boltovskoy, ed.). INIDEP, Mar del Plata, p. 383-441.

AMARAL, F.D. 1997. Milleporidae (Cnidaria, Hydrozoa) do litoral Brasileiro. PhD Thesis, Universidade de São Paulo, São Paulo.

ANDRADE, L.P. 2001. Aspectos da biologia e do ciclo de vida de Aglaophenia latecarinata (Cnidaria, Hydrozoa, Aglaopheniidae). MSc. Dissertation, Universidade de São Paulo, São Paulo.

ANDRADE, L.P. \& MIGOTTO, A.E. 1996. O ciclo de vida de Hebella furax (Cnidaria, Hydrozoa, Lafoeidae). In Resumos do III Simpósio sobre oceanografia. IOUSP, São Paulo, p. 179.

ANDRADE, L.P. \& MIGOTTO, A.E. 1997. Is there a link 
between Hebella hydroids (Hydrozoa, Lafoeidae) and Staurodiscus medusae (Hydrozoa, Laodiceidae)? In Boletim de Resumos Expandidos VII COLACMAR, v. 1, Santos, p. 35-36.

BELéM, M.J.C., CASTRO, C.B. \& ROHLFS C. 1982. Notas sobre Solanderia gracilis Duchassaing \& Michelin, 1846, do Parcel de Abrolhos, BA. Primeira ocorrência de Solanderiidae (Cnidaria, Hydrozoa) no litoral brasileiro. An. Acad. brasil. Ciênc. 54(3): 585588.

BOUILLON, J. \& GROHMANN, P.A. 1990. Pinushydra chiquitita gen. et sp. nov. (Cnidaria, Hydrozoa). Cah. Biol. Mar. 31: 291-305.

BOUILLON, J. \& GROHMANN, P.A. 1994. A new interstitial stolonal hydroid: Nannocoryne gen. nov. mammylia sp. nov. (Hydroidomedusae, Anthomedusae, Corynidae). Cah. Biol. Mar. 35: 431439.

BRANNER, J.C. 1904. The stone reefs of Brazil, their geological and geographical relations with a chapter on the coral reefs. Bull. Mus. comp. Zool. Harv. 54: 1285.

CALDER, D.R. 1988. Shallow-water hydroids of Bermuda: The Athecatae. Life Sci. Contri. R. Ontario Mus. 148: 1-107.

CALDER, D.R. 1991. Shallow-water hydroids of Bermuda: The Thecatae, exclusive of Plumularioidea. Life Sci. Contri. R. Ontario Mus. 154: 1-140.

CALDER, D.R. 1997. Shallow-water hydroids of Bermuda: supefamily Plumularioidea Life Sci. Contri. R. Ontario Mus. 161: 1-85.

CALDER, D.R. \& MAÿAL, E.M. 1998. Dry season distribution of hydroids in a small tropical estuary, Pernambuco, Brazil. Zool. Verh. Leiden 323: 69-78.

CARVALHO, J.P. 1943. Ocorrência do gênero Liriope no plancton marinho de Santos e São Vicente. Bol. Ind. Anim. 148: 105-111.

CASTRO, C.B., ECHEVERRÍA, C.A., PIRES, D.O., MASCARENHAS, B.J.A. \& FREITAS, S.G. 1995. distribuição de Cnidaria e Echinodermata no infralitoral de costões rochosos de Arraial Cabo, Rio de Janeiro, Brasil. Rev. Brasil. Biol. 55(3): 471-480.

CASTRO, C.B. \& PIRES, D.O. 2001. Brazilian coral reefs: what we already know and what is still missing. Bull. mar. Sci. 69(2): 357-371.

CASTRO, C. B. \& SEGAL, B. 2001. The itacolomis: large and unexplored reefs at the arrival point of the first Europeans in Brazil. Coral Reefs 20: 18.

CASTRO, C.B., SILVEIRA, F.L. \& MORANDINI, A.C. 1998. Primeiro registro de uma medusa de Scyphozoa para o Parque Nacional Marinho dos Abrolhos, BA, através de documentação em video (H18). In XXII Congresso Brasileiro de Zoologia. SBZ, Recife, PE, p. 7.

CORNELIUS, P.F.S. \& SILVEIRA, F.L. 1997. Recent observations on the Brazilian scyphomedusa fauna. In Boletim de Resumos Expandidos VII
COLACMAR, v. 1, Santos, p 192-194.

CORREIA, K.V. 1985. Ocorrência de Bougainvillia trinema (von Lendenfeld, 1884) (AnthomedusaeBougainvilliidae) para o Atlântico. In Resumos do XII Congresso Brasileiro de Zoologia. SBZ, Campinas, p.15.

DAWSON, M.N. \& JACOBS, D.K. 2001. Molecular evidence for cryptic species of Aurelia aurita (Cnidaria, Scyphozoa). Biol. Bull. 200: 92-96.

DIAS, L. 1994. Siphonophora (Cnidaria, Hydrozoa) da região compreendida entre Cabo Frio, $\mathrm{RJ}\left(23^{\circ} \mathrm{S}\right)$ e Cabo de Santa Marta Grande, SC $\left(29^{\circ} \mathrm{W}\right)$. PhD Thesis, Universidade de São Paulo, São Paulo.

ECHEVERRíA, C.A., PIRES, D.O., MEDEIROS, M.S. \& CASTRO, C.B. 1997. Cnidarians of the Atol das Rocas, Brazil. Proc. 8th International Coral Reef Symposium, Panama, 1: 443-446.

ESCHSCHOLTZ, F. 1829. System der Acalephen. Eine ausführliche Beschreibung aller medusenartigen Strahltiere. Berlin, 1-190.

ESTON, V.R., MIGOTTO, A.E., OLIVEIRA FILHO, E.C., RODRIGUES, S.A. \& FREITAS, J.C. 1986. Vertical distribution of benthic marine organisms on rocky coasts of the Fernando de Noronha Archipelago (Brazil). Bolm Inst. Oceanogr., S. Paulo 34:37-53.

FREITAS, J.C. SCHIOZER, W.A. \& MALPEZZI, E.L.A. 1995. A case of envenoming by Portuguese man-ofwar from the Brazilian coast. Toxicon 33(7): 859861.

GOY, J. 1979. Campagne de la Calypso au large des côtes atlantiques de l'Amérique du Sud (1961-1962) - 35. Méduses. Resul. scient. camp.Calypso 11: 263-296.

GRAVIER-BONNET, N. \& MIGOTTO, A.E. 2000. Gonangium development and medusoid of Nemalecium lighti (Hargitt, 1924) (Cnidaria: Hydrozoa, Haleciidae). Sci. Mar. 64(supl. 1): $207-$ 213.

GROHMANN, P.A., SOUZA, M.M. \& NOGUEIRA, C.C. 1997. Hydroids from the vicinity of a large industrial area in Vitória, Espírito Santo, Brazil. Proceedings of the VI International Conference on Coelenterate Biology, Leiden, The Netherlands: 227-232.

GROHMANN, P.A., M.P. MAGALHãES \& Y.M. HIRANO 1999. First record of the order Stauromedusae (Cnidaria, Scyphozoa) from the tropical Soutwestern Atlantic, with a review of the distribution of Stauromedusae in the southern hemisphere. Species Diversity 4: 381-388.

HADDAD JR., V., CARDOSO, J.L.C. \& SILVEIRA, F.L. 2001. Seabather's eruption: report of five cases in Southeast region of Brazil. Rev. Inst. Med. Trop. SPaulo 43(3): 171-172.

HADDAD, M.A. 1992. Hidróides (Cnidaria, Hydrozoa) de costões rochosos do litoral sul do Estado do Paraná. PhD Thesis, Universidade de São Paulo, São Paulo.

HADDAD, M.A. \& NAKATANI, K. 1996. Primeiro registro de Cordylophora caspia (Pallas, 1771) 
(Cnidaria, Hydroida) em águas continentais brasileiras. In XIV Congresso Brasileiro de Zoololgia., SBZ, Porto Alegre, p.10.

HADDAD, M.A., SILVA, J.D. da \& OLIVEIRA, C.C. de 2000. Hidróides (Cnidaria, Hydrozoa) epifíticos em Sargassum cymosum C.Agarth, 1820 (Phaeophyta, Fucales) da Ponta das Garoupas, Bombinhas, Santa Catarina. In XXIII Congreso Brasileiro de Zoologia. SBZ, Cuiabá, p.42.

HAECKEL, E. 1880. Das system der medusen. I, 2: System der Acraspeden. Zweite Halfte des System der Medusen. Gustav Fischer, Jena, 361-672.

HARTT, C. F. 1868. A vacation trip to Brazil. Amer. Natur. 1: 642-651.

HARTT, C. F. 1869. The cruise of the Abrolhos. Amer. Natur. 2: 85-93.

HETZEL, B. \& CASTRO, C.B. 1994. Corais do Sul da Bahia. Rio de Janeiro, Nova Fronteira.

HORTA, P.A., MORANDINI, A.C., BUCCHMANN, F. \& OLIVEIRA, E.C. 2001. Macrobentos do Parcel do Carpinteiro - um desafio para o levantamento da biodiversidade do infralitoral brasileiro. In Anais da XIV Semana Nacional de Oceanografia Oceanografia e Sociedade: um desafio à teoria e à prática. FURG, Rio Grande, resumo 126.

HUDSON, M.M.F., AMARAL, F.D., COURA, M.F. 1999 Atualização do levantamento de cnidários bentônicos do Parque Estadual Marinho do Parcel do Manuel Luiz, MA. In Resumos do XII Encontro de Zoologia do Nordeste, p. 180.

JäDERHOLM, E. 1903. Aussereuropäische hydroiden in Schwedischen Reichsmuseum. Ark. Zool. 1: 259312.

JäDERHOLM, E. 1920. On some exotic hydroids in the Swedish Zoological State Museum. Ark. Zool. 13(3): $1-11$

KELMO, F. \& DE SANTA-ISABEL, L.M. 1998. The athecatae hydroids (Cnidaria, Hydrozoa) from Northern Bahía, Brazil. Rev. Biol. Trop. 46 (supl.5): 61-72.

KRAMP, P.L. 1951. Hydrozoa and Scyphozoa. Rep. Swed. Deep-Sea Exped., Zoology 2(10): 121-127.

KRAMP, P.L. 1957. Hydromedusae from the Discovery collections. Discovery Rep. 29: 1-128.

KRAMP, P.L. 1959. The Hydromedusae of the Atlantic Ocean and adjacent waters. Dana-Rep. 46: 1-283.

KRAMP, P.L. 1961. Synopsis of the medusae of the world. J. mar. biol. Ass. U.K. 40: 1-469.

LABOREL, J. 1969a. Madréporaires et Hydrocoralliaires récifaux des côtes brésiliennes. Systematique, ecologie, repartition verticale et geographie. Ann. Inst. Oceanogr., Paris 47: 171-229

LABOREL, J. 1969b. Les peuplements de madréporaires des côtes tropicales du Brésil. Ann. Univ. Abidjan Ecologie, series E II(3): 1-260.

LELOUP, E. 1935. Contribution à la répartition de Tetraplatia volitans (Busch). Bull. m. r. hist. nat. Belgiq 11(4): 1-7.
LESSON, R.P. 1830. Voyage autour du monde, exécuté par ordre du roi sur la corvette La Coquille, pendant les anées 1822-1825. Zoologie t.2(2): 1-135.

LINDNER, A. 2000. Redescrição e ciclo de vida de Clytia gracilis e Clytia linearis (Cnidaria, Hydrozoa, Campanulariidae). MSc. Dissertation, Universidade de São Paulo, São Paulo.

LINDNER, A. \& CALDER, D.R. 2000. Campanularia noliformis McCrady, 1859 (currently Clytia noliformis; Cnidaria, Hydrozoa): proposed conservation of the specific name by the designation of a neotype. Bull. Zool. Nom. 57, 140-143.

LINDNER, A. \& MIGOTTO, A.E. 1998. O ciclo de vida de Clytia noliformis (Cnidaria, Hydrozoa, Campanulariidae). In XXII Congresso Brasileiro de Zoologia. SBZ, Recife, p. 6.

LINDNER, A. \& MIGOTTO, A.E. 2001. Merotrichous isorhiza, a new nematocyst in the Campanulariidae (Cnidaria; Hydrozoa), and its relevance for the classification of cnidae. Proc. biol. Soc. Wash. 114(4): 825-832.

MARQUES, A.C. 1993. Sistemática dos Eudendriidae L. Agassiz, 1862 (Cnidaria, Hydrozoa) do litoral Paulista. São Paulo. MSc. Dissertation, Universidade de São Paulo, São Paulo.

MARQUES, A.C. 1995. Eudendrium pocaruquarum n. sp. (Hydrozoa, Eudendriidae) from the southeastern coast of Brazil, with remarks on taxonomic approaches to the family Eudendriidae. Contr. Zool. 65(1): 35-40.

MARQUES, A.C. 2001. O gênero Eudendrium (Cnidaria, Hydrozoa, Anthomedusae) no Brasil. Pap. Av. Zool. 41(22): 329-405.

MARQUES, A.C., HADDAD, V. \& MIGOTTO, A.E. 2002. Envenomation by a benthic Hydrozoa (Cnidaria): the case of Nemalecium lighti (Haleciidae). Toxicon 40: 213-215.

MARQUES, A.C., MORANDINI, A.C. \& PINTO, M.M. 1997. Cnidome of Chiropsalmus quadrumanus (Cnidaria, Cubozoa) from Brazil. In Boletim de Resumos Expandidos VII COLACMAR, Santos, v. 2, p 136-138.

MARQUES, A.C. \& MORETZSOHN, F. 1995. Substrata used by Pteria colymbus (Röding, 1798) (Bivalvia: Pteriidae) for attachment. Publção. esp. Inst.oceanogr. SPaulo 11: 197-201.

MAÿAL, E.M. 1983. Distribuição de hidróides (Hydrozoa, Thecata) na costa do estado de Pernambuco, Brasil. Bolm. Zool. Univ. S. Paulo 8: 1-13.

MAYER, A.G. 1910. The medusae of the world. Vol III. The scyphomedusae. Carnegie Institution, Washington D.C.

MIANZAN, H.W. \& CORNELIUS, P.F.S. 1999. Cubomedusae and Scyphomedusae. In South Atlantic Zooplankton (D. Boltovskoy ed.). Backhuys Publishers, Leiden, Vol. 1, p.513-559.

MIANZAN, H.W. \& GUERRERO, R.A. 2000. Environmental patterns and biomass distribution of 
gelatinous macrozooplankton. Three study cases in the South-western Atlantic Ocean. Sci. Mar. 64(Supl. 1): 215-224.

MIGOTTO, A.E. 1996. Benthic shallow-water hydroids (Cnidaria, Hydrozoa) of the coast of São Sebastião, Brazil, including a checklist of Brazilian hydroids. Zool. Verh. Leiden 306: 1-125.

MIGOTTO, A.E. 1997. Aspects of the reproduction of Dentitheca bitentata (Cnidaria, Hydrozoa): a monoic plumularid that releases medusoids. In Boletim de Resumos Expandidos VII COLACMAR, v. 2, Santos, p. $170-171$.

MIGOTTO, A.E. 1998. The life cycle of Sertularia marginata Kirchenpauer, 1864 (Cnidaria: Hydrozoa): a medusoid-producing sertulariid. J. nat. Hist. 32: 1-12.

MIGOTTO, A.E. \& ANDRADE, L.P. 2000. The life cycle of Hebella furax (Cnidaria: Hydrozoa): a link between a lafoeid hydroid and a laodiceidae medusae. J. nat. Hist. 34: 1871-1888.

MIGOTTO, A.E. \& CALDER, D.R. 1998. Sertularia vervoorti (Hydrozoa: Sertulariidae), an undescribed species of hydroid from Brazil. Zool. Verh. Leiden 323:169-174.

MIGOTTO, A.E. \& MARQUES, A.C. 1999a. Hydroid and medusa stages of the new species Ectopleura obypa (Cnidaria: Hydrozoa: Tubulariidae) from Brazil. Proc. biol. Soc. Wash. 112(2):303-312.

MigotTO, A.E. \& MARQUES, A.C. 1999 b. Redescription of Dentitheca bidentata (Hydrozoa, Leptomedusae, Plumulariidae) with notes on its life cycle. J. nat. Hist 33: 949-960.

MIGOTTO, A.E., MARQUES, A.C. \& FLYNN, M.N. 2001. Seasonal recruitment of hydroids (Cnidaria) on experimental panels in the São Sebastião Channel, Southeastern Brazil. Bull. Mar. Sci. 68: 287-298.

MIGOTTO, A.E. \& SILVEIRA, F.L. 1987. Hidróides (Cnidaria, Hydrozoa) do litoral sudeste e sul do Brasil: Halocordylidae, Tubulariidae e Corymorphidae. Iheringia. sér.Zool. 66: 3-32.

MIGOTTO, A.E. \& TIAGO, C. 1999. Biodiversidade do Estado de São Paulo: Síntese do conhecimento no final do século XX. Parte 3: Invertebrados marinhos. FAPESP, São Paulo.

MIGOTTO, A.E. \& VERVOORT, W. 1998. Redescription of Sertularia notabilis Fraser, 1947 (Sertulariidae, Hydrozoa). Zool. Med. Leiden 72: 89-100.

MORANDINI, A.C. \& MARQUES, A.C. 1997. "Morbakka" syndrome: frst report of envenomation by Cubozoa (Cnidaria) in Brazil. In Boletim de Resumos Expandidos VII COLACMAR, Santos, v. 2, p.188-189.

MORANDINI, A.C. \& SILVEIRA, F.L. 2001a. Sexual reproduction of Nausithoe aurea (Scyphozoa, Coronatae). Gametogenesis, egg release, embryonic development, and gastrulation. Sci. Mar. 65(2): 139149.

MORANDINI, A.C. \& SILVEIRA, F.L. 2001b. New observations and new record of Nausithoe aurea (Scyphozoa, Coronatae). Pap. Av. Zool. 41(27): 519527.

MOREIRA, M.G.B.S. 1961. Sobre Mastigias scintillae sp. nov. (Scyphomedusae, Rhizostomeae) das costas do Brasil. Bolm Inst. Oceanogr. S Paulo 11(2): 5-29.

MOREIRA, G.S. 1973. On the diurnal vertical migration of hydromedusae off Santos, Brazil. Publs Seto mar. biol. Lab. 20: 537-566.

MOREIRA, G.S. 1975. Sobre duas Leptomedusae do litoral do estado de São Paulo. Ciênc. Cult., S. Paulo 27(5): 556-558.

MOREIRA, G.S., LEITE L.R. \& NIPPER, M.G. 1978. Notes on Dipurena reesi Vannucci 1956 (Hydrozoa, Corynidae) with a description of an unusual method of asexual reproduction. Bolm Fisiol. Anim., Univ. S Paulo 2: 159-164.

MOREIRA, G.S., NIPPER, M.G. \& LEITE, L.R. 1979. On Stylactis hooperi Sigerfoos, 1899 (Hydrozoa, Hydractiniidae, a new addition to the fauna of southern Brazil. Proc. Internat. Symp. Mar. Biogeogr. Evolut. Southern Hemisph. New Zealand 2: 679-689.

MOREIRA, G. S. \& YAMASHITA, C. 1972. Aglauropsis kawari (Limnomedusae: Olindiasidae), a new species from the South Atlantic. Mar. Biol. 14(3): 271-274.

MOURA, R.L., MARTINS-RODRIGUES, M.C., FRANCINI-FILHO, R.B., SAZIMA, I. 1999. Unexpected richness of reef corals near the Southern Amazon River mouth. Coral Reefs 18: 170.

MüLLER, F. 1859a. Polypen und Quallen von Santa Catharina. Die Formwandlungen der Liriope catharinensis n.sp. Archiv für Naturgeschichte 1: 310-321. In Fritz Müller: Werke, Briefe und Leben. Erster Band: Gesammelte schriften sowiet sie bereits früher in druck erschienen sind (A Möller ed., 1915) Jena, vol. 1, p. 93-100.

MüLLER, F. 1859b. Zwei neue Quallen von Santa Catharina. Tamoya haplonema und quadrumana. Abhandlungen der Naturforschenden Gesellschaft in Halle 5: 1-12. In In Fritz Müller: Werke, Briefe und Leben. Erster Band: Gesammelte schriften sowiet sie bereits früher in druck erschienen sind (A Möller ed., 1915) Jena, vol. 1, p. 85-92.

MÜLLER, F. 1861a. Polypen und Quallen von Santa Catharina. Olindias sambaquiensis n.sp. Archiv für Naturgeschichte 1: 312-319. In Fritz Müller: Werke, Briefe und Leben. Erster Band: Gesammelte schriften sowiet sie bereits früher in druck erschienen sind (A Möller ed., 1915) Jena, vol. 1, p. 132-136.

MÜLLER, F. 1861b. Cunina Köllikeri n.sp. Beitrag zur Naturgeschichte der Aeginiden. Archiv für Naturgeschichte 1: 42-52. In Fritz Müller: Werke, Briefe und Leben. Erster Band: Gesammelte schriften sowiet sie bereits früher in druck erschienen sind (A Möller ed., 1915) Jena, vol. 1, p. 116-122.

MüLLER, F. 1865. Über die Randbläschen der Hydroidquallen. Arch. mikr. Anat. 1: 143-147.

MüLLER, F. 1883.Drymonema an der küste von Brasilien. 
Zool. Anz. 6: 220-222. In Fritz Müller: Werke, Briefe und Leben. Erster Band: Gesammelte schriften sowiet sie bereits früher in druck erschienen sind (A Möller ed., 1915) Jena, vol. 1, p. 999-1000.

NARCHI, W. \& HEBLING, N.J. 1975. The life cycle of the commensal hydromedusa Eutima sapinhoa n. sp. Mar. Biol. 30: 73-78.

NAVAS-PEREIRA, D. 1980. Hydromedusae of the Bay of Sepetiba (Rio de Janeiro, Brazil). Revta bras. Biol. 40(4):817-824.

NAVAS-PEREIRA， D. 1981. Distribuição das hidromedusas (Cnidaria, Hydrozoa) na região da plataforma continental do Rio Grande do Sul. In Seminários de Biologia Marinha, São Paulo. Academia Brasileira de Ciências. p. 221-276

NAVAS-PEREIRA, D. 1984a. New record of budding in Zanclea costata (Anthomedusae, Zancleidae) Dusenia 14(3): 89-93.

NAVAS-PEREIRA, D. 1984b. On the morphological variability of Phialucium carolinae (Mayer, 1900) (Leptomedusae, Phialuciidae). Dusenia 14(2): 51-53.

NOGUEIRA, C.C., GROHMANN, P.A. \& DA SILVA, V.M.A.P. 1997. Hydroids from the vicinity of a nuclear power plant site (CNAAA-Unidade I) at Angra-dos-Reis, Rio de Janeiro, southeastern Brazil. Proceedings of the VI International Conference on Coelenterate Biology, Leiden, The Netherlands: 365369.

NUTTING, C.C. 1900. American hydroids. Part I. The Plumulariidae. Spec. Bull. U.S. natn. Mus. 4: 1-285.

NUTTING, C.C. 1904. American hydroids. Part I. The Sertulariidae. Spec. Bull. U.S. natn. Mus. 4: 1-325.

OLIVEIRA, L.P.H. 1949. Relatório sôbre as excursões científicas feitas a bordo do Navio Hidrográfico Rio Branco etc. Mem. Inst. Osw. Cruz 47: 87-96.

OLIVEIRA, L.P.H. 1950. Levantamento biogeográfico da Baía de Guanabara. Mem. Inst. Osw. Cruz 48: 363 391.

OLIVEIRA, O.M.P. 2000. História Natural de Eudendrium glomeratum (Cnidaria, Anthomedusae, Eudendriidae) do canal de São Sebastião, SE Brasil. Bachelorship monograph. Universidade de São Paulo, Ribeirão Preto.

OLIVEIRA, O.M.P., MARQUES, A.C. \& MIGOTTO, A.E. 2000. Morphometric patterns of two fouling Eudendrium spp. (Hydrozoa, Anthomedusae, Eudendriidae) from São Sebastião (SP, SE Brazil). Braz. Arch. Biol. Tech. 43(5):519-523.

PANTIN, C.F.A. \& DIAS, M.V. 1952. Rhythm and afterdischarge in medusae. An. Acad. bras. Ciênc. 24(4): 351-364.

PARANAGUÁ, M.N. 1963. Sobre uma nova ocorrência de Blackfordia virginica Mayer, 1910 e Ostrumovia inkermanica Hadzi (1928) (Hydromedusae). Trab. Inst. oceanogr. Univ. Recife 5-6: 141-145.

PIRES, D.O. 1985. Observações biológicas e taxonômicas em Cladonema sp. do Rio de Janeiro (Cnidaria, Hydroida). IN Resumos do XII Congresso Brasileiro de Zoologia. SBZ, UNICAMP, p. 10.

PIRES, D.O., CASTRO, C.B., MIGOTTO, A.E. \& MARQUES, A.C. 1992. Cnidários bentônicos do Arquipélago de Fernando de Noronha, Brasil. Bolm Mus. nac. Rio de Janeiro, Zool. 354: 1- 21.

PUGH, P.R. 1999. Siphonophorae. In South Atlantic Zooplankton (D. Boltovskoy, ed.). Backhuys Publishers, Leiden, p. 467-511.

RAMÍREZ, F.C. \& ZAMPONI, M.O. 1981. Hydromedusae. In Atlas del zooplancton del Atlántico sudoccidental y metodos de trabajo con el zooplancton marino (D. Boltovskoy, ed). INIDEP, Mar del Plata, p 443-469.

RITCHIE, J. 1909. Supplementary report on the hydroids of the Scottish National Antartic Expedition. Trans. R. Soc. Edinb. 47: 65-101.

ROSSO, S. \& MARQUES, A.C. 1997. Is there any conspicuous geographical pattern in intertidal hydrozoan distribution along the coast of São Paulo State, Southeastern Brazil ? Proceedings of the VI International Conference on Coelenterate Biology, Leiden, Netherlands: 415-422.

SALLES, V. 1994. Guia do Meio Ambiente Litoral de Alagoas. Instituto do Meio Ambiente \& Deutsche Gesellschaft für technische Zusammenarbeit [Projeto IMA-GTZ], Alagoas.

SILVEIRA, F.L. \& BOSCOLO, H.K. 1996. Primeira ocorrência de Cordylophora caspia (Pallas, 1771) (Hydrozoa, Clavidae) para o Estado de São Paulo. In Resumos XIV Congresso Brasileiro de Zoologia. SBZ, Porto Alegre, p.10.

SILVEIRA, F.L. \& CORNELIUS, P.F.S. 2000. Novas observações sobre medusas (Cnidaria, Scyphozoa, Rhizostomeae) no nordeste e sul do Brasil. Acta Biol. Leopold. 22(1): 9-18.

SILVEIRA, F.L., CORNELIUS, P.F.S., SCHLENZ, E., MORANDINI, A.C. \& MONTEIRO, G.R.C. 1998. "Macromedusas" (Cnidaria, Scyphozoa e Cubozoa) do Canal de São Sebastião. In XIII Simpósio de Biologia Marinha. CEBIMar USP, São Sebastião,p. 66.

SILVEIRA, F.L. \& MIGOTTO, A.E. 1984. Serehyba sanctisebastiani n.gen., n.sp. (Hydrozoa, Tubulariidae), symbiont of a gorgonian octocoral from the southeast coast of Brazil. Bijdr. Dierk. 54(2):231-242

SILVEIRA, F.L. \& MIGOTTO, A.E. 1991. The variation of Halocordyle disticha (Cnidaria, Athecata) from the Brazilian coast: an environmental indicator species? Hydrobiologia 216/217: 437-442.

SILVEIRA, F.L. \& MIGOTTO, A.E. 1992. Rediscovery of Corymorpha januarii Steenstrup, 1854 (Hydrozoa, Corymorphidae) on the southeastern and southern coasts of Brazil. Steenstrupia 18(4): 81-89.

SILVEIRA, F.L.; MIGOTTO, A.E. \& MORANDINI, A.C. 2000. Primeira ocorrência no litoral paulista da cifomedusa Cassiopea xamachana Bigelow, 1892 (Rhizostomeae) através do cultivo e observações dos 
cifístomas, estróbilos, éfiras e medusas. In XV Simpósio de Biologia Marinha. CEBIMar-USP, São Sebastião, p. 66.

SILVEIRA, F.L., MIGOTTO, A.E. \& VIEITAS, C.F. 1988. Staurocladia Hartlaub, 1917 (Hydroida, Anthomedusae, Eleutheriidae) do infralitoral de Itaipava, ES, ocorrência nova. In Resumos do XV Congresso Brasileiro de Zoologia. SBZ, Curitiba, $\mathrm{p}$ 22.

SILVEIRA，F.L. \& MORANDINI, A.C. 1996. Stephanoscyphistoma corniformis (Komai, 1936) (Cnidaria, Scyphozoa, Coronatae) from the north coast of São Paulo, Brazil; redescription and the first record in the western South Atlantic. Semina 17(2):137-145.

SILVEIRA, F.L. \& MORANDINI, A.C. 1997. Nausithoe aurea n. sp. (Scyphozoa, Coronatae, Nausithoidae), a species with two pathways of reproduction after strobilation: sexual and asexual. Contr. Zool. 66(40):235-246.

SILVEIRA, F.L. \& MORANDINI, A.C. 1998a. Asexual reproduction in Linuche unguiculata (Swartz, 1788) (Scyphozoa: Coronatae) by planuloid formation through strobilation and segmentation. Proc. biol. Soc. Wash. 111(4):781-794.

SILVEIRA, F.L. \& MORANDINI, A.C. 1998b. New observations on dormancy mechanisms in Linuche unguiculata (Swartz, 1788) (Scyphozoa: Coronatae). Bol. Mus. Nac., N.S., Zool. 393:1-7, figs.1-2.

STECHOW, E. 1912. Hydroiden der Münchener Zoologischen Staatssammlung. Zool. Jb., Syst. 32(4): 333-378.

STECHOW, E. 1919. Zur Kenntis der Hydroidenfauna des Mittelmeeres, Amerikas und anderer Gebiete, nebst Angaben über einige Kirchenpauer'sche Typen von Plumulariden. Zool. Jb., Syst. 42(1): 1-172

STEENSTRUP, J.J.S. 1854. En ny og tropisk Art af Smaagoplernes Ammeslaegt: Corymorpha Sars (Corym. Januarii Stp). Vidensk. Meddr. danks maturh. Foren. 1-3: 46-48.

THIEL, M.E. 1935. Die Beziedlung der Südatlantischen Oceans mit Hydromedusen. Wiss. Erg. dt. Atl. Exped. Meteor 12(2): 32-100.

THIEL, M.E. 1938. Die Leptolinae der "Meteor" Expedition in systematischer Betrachtung (I. Anthomedusae). Zool. Anz. 121:289-303.

TOTTON, A.K. 1926. Note on a rare Atlantic hydroid. Ann. Mag. nat. Hist. 9: 210-212.

TRONOLONE， V.B. 2001 Hidromedusas (Cnidaria, Hydrozoa) do canal de São Sebastião, SP. MSc. Dissertation, Universidade de São Paulo, São Paulo.

VANHöFFEN, E. 1888. Untersuchungen über semästome und rhizostome Medusen. Biblioth. Zool. 1(3): 52p.

VANHöFFEN, E. 1908. Die Lucernariden und Skyphomedusen der Deustschen SüdpolarExpedition 1901-1903. Dtsch Südpolar-Exped.10 (Zoologie 2): 25-49.

VANHöFFEN, E. 1913. Die Craspedoten Medusen des
"Vetor Pisani". Zoologica 67:1-34.

VANNUCCI-MENDES, M. 1944. Sôbre a larva de Dibothriorhynchus dinoi, sp. n. parasita dos Rhizostomata. (Cestoda Tetrarhynchidea). Arq. Mus. Paran. 4: 47-82.

VANNUCCI-MENDES, M. 1946. Hydroida Thecaphora do Brasil. Arq. Zool. Estado São Paulo 4: 535-538.

VANNUCCI-MENDES, M. 1948. On Vallentinia gabriellae, n. sp. (Limnomedusae). Bolm Fac. Filos. Ciênc. Univ. S Paulo, Zool. 13: 73-91.

VANNUCCI, M. 1949. Hydrozoa do Brasil. Bolm Fac. Filos. Ciênc. Univ. S Paulo 99(14): 219-266.

VANNUCCI, M. 1950. Resultados científicos do Cruzeiro do "Baependi" e do "Vega" a Ilha da Trindade. Hydrozoa. Bolm Inst. oceanogr., S Paulo 1(1): 81-96.

VANNUCCI, M. 1951a. Hydrozoa e Scyphozoa existentes no Instituto Paulista de Oceanografia. I. Bolm Inst. oceanogr., S Paulo 2(1): 67-98.

VANNUCCI, M. 1951b. Distribuição dos Hidrozoa até agora conhecidos nas costas do Brasil. Bolm Inst. oceanogr., S Paulo 2(1): 105-124.

VANNUCCI, M. 1954. Hydrozoa e Scyphozoa existentes no Instituto Oceanográfico. II. Bolm Inst. oceanogr., S Paulo 5(1-2): 95-149.

VANNUCCI, M. 1956. Biological notes and description of a new species of Dipurena (Hydrozoa, Corynidae). Proc. Zool. Soc. Lond. 127:479-487.

VANNUCCI, M. 1957a. On brazilian hydromedusae and their distribution in relation to different water masses. Bolm Inst. oceanogr., S Paulo 8(1-2): 23-109.

VANNUCCI, M. 1957b. Distribuição de Scyphozoa nas costas do Brasil. An. acad. bras. Ciênc. 29(4): 593598.

VANNUCCI, M. 1960. On the young stage of Eucheilota duodecimalis (Leptomedusae). An. acad. bras. Ciênc. 32(3/4): 395-397.

VANNUCCI, M. 1963. On the ecology of Brazilian Medusae at $25^{\circ}$ lat. S. Bolm Inst. oceanogr., S Paulo 13(1):143-184.

VANNUCCI, M. \& MOREIRA, G.S. 1966. New species and new record of Anthomedusae from southern Brazil. Bolm. Inst. oceanogr., S Paulo 15: 85-90.

VANNUCCI, M. \& REES, W.J. 1961. A revision of the genus Bougainvillia (Anthomedusae). Bolm Inst. oceanogr., S Paulo 11(2): 57-100.

VANNUCCI, M. \& RIBEIRO, L.C. 1955. O ciclo reprodutivo de Clytia cylindrica L. Agass., 1862 (Hydrozoa: Campanulariidae). Dusenia 6(3/4): 6980.

VERRILL, A.E. 1868. Notes on Radiata in the Museum of Yale College, with descriptions of new genera and species. 4. Notice of corals and echinoderms collected by C. F. Hartt, at the Abrolhos Reefs, Province of Bahia, Brazil, 1867. Trans. Conn. Acad. Arts Sci., New Haven 1: 351-371.

VERVOORT, W. 1946. Exotic hydroids in the collections of the Rijksmuseum van Natuurlijke Historie and the Zoological Museum at Amsterdam. Zool. Meded., 
Leiden 26 (1-4): 287-351.

WEERDT, W.H. 1984. Taxonomic characters in Caribbean Millepora species (Hydrozoa, Coelenterata). Bijdr. Dierk. 54(2): 243-262.
Title: Checklist of the Cnidaria Medusozoa of Brazil

Authors: Alvaro E. Migotto,Antonio C. Marques,André C. Morandini,Fábio L. da Silveira

Biota Neotropica, Volume 2( number 1) 2002 http://www.biotaneotropica.org.br/v2n1/pt/fullpaper?bn0 $1102012002+$ en

Date Received: 02/25/ 2002

Revised: 04/15/ 2002

Accepted:04/19/ 2002

ISSN 1676-0611 
Table 1. Species of Medusozoa recorded from Brazilian waters. See abbreviations in the text

\begin{tabular}{|c|c|c|c|c|}
\hline Species & Region & State & Reference & Stage \\
\hline \multicolumn{5}{|l|}{ Class Hydrozoa } \\
\hline \multicolumn{5}{|l|}{ Subclass Anthomedusae } \\
\hline \multicolumn{5}{|l|}{ Order Capitata } \\
\hline \multicolumn{5}{|l|}{ Family Asyncorynidae } \\
\hline Asyncoryne ryniensis Warren, 1908 & $\mathrm{SE}$ & SP & Migotto 1996 & $\mathrm{P} / \mathrm{M}$ \\
\hline \multicolumn{5}{|l|}{ Family Cladocorynidae } \\
\hline Cladocoryne floccosa Rotch, 1871 & SE, NE & $\mathrm{SP}, \mathrm{ES}, \mathrm{PE}$ & $\begin{array}{l}\text { Migotto 1996, Grohmann et al. 1997, } \\
\text { Calder \& Maÿal } 1998\end{array}$ & $\mathrm{P}$ \\
\hline \multicolumn{5}{|l|}{ Family Cladonematidae } \\
\hline Cladonema radiatum Dujardin, 1843 & SE & SP & Migotto 1996 & $\mathrm{P} / \mathrm{M}$ \\
\hline Cladonema sp. & SE & RJ & Pires 1985 & $\mathrm{P} / \mathrm{M}$ \\
\hline \multicolumn{5}{|l|}{ Family Cladosarsiidae } \\
\hline Cladosarsiidae sp. indet. 1 & SE & SP & Tronolone 2001 & $\mathrm{M}$ \\
\hline \multicolumn{5}{|l|}{ Family Corymorphidae } \\
\hline Corymorpha forbesi (Mayer, 1894) & S, SE & $\mathrm{RS}, \mathrm{RJ}$ & Navas-Pereira 19801981 & $\mathrm{M}$ \\
\hline Corymorpha furcata (Kramp, 1948) & S, SE, NE & $\begin{array}{l}\text { no specific } \\
\text { record }\end{array}$ & Ramirez \& Zamponi 1981 & M \\
\hline Corymorpha gracilis (Brooks, 1882) & S, SE & RS, SP, RJ & $\begin{array}{l}\text { Vannucci 1957a 1963, Moreira 1973, Goy } \\
\text { 1979, Navas-Pereira } 19801981, \\
\text { Tronolone 2001 }\end{array}$ & $M$ \\
\hline Corymorpha januarii Steenstrup, 1854 & $\mathrm{~S}, \mathrm{SE}$ & $\mathrm{SC}, \mathrm{SP}, \mathrm{RJ}$ & $\begin{array}{l}\text { Steenstrup 1854, Silveira \& Migotto 1992, } \\
\text { Tronolone } 2001\end{array}$ & $\mathrm{P} / \mathrm{M}$ \\
\hline Eucodonium brownei Hartlaub, 1907 & $\mathrm{~S}, \mathrm{SE}$ & $\begin{array}{l}\text { no specific } \\
\text { record }\end{array}$ & Vannucci 1957a 1963, Moreira 1973 & M \\
\hline \multicolumn{5}{|l|}{ Family Corynidae } \\
\hline Coryne pusilla Gaertner, 1774 & S, SE & PR, RJ, ES & $\begin{array}{l}\text { Haddad 1992, Grohmann et al. 1997, } \\
\text { ?Nogueira et al. } 1997\end{array}$ & $\mathrm{P}$ \\
\hline Dicodonium punctatum Vanhöffen, 1911 & $\mathrm{~N}$ & $\begin{array}{l}\text { no specific } \\
\text { record }\end{array}$ & Kramp 1961 & $\mathrm{M}$ \\
\hline Dipurena reesi Vannucci, 1956 & $\mathrm{SE}$ & SP, ES & $\begin{array}{l}\text { Vannucci 1956, Moreira et al. 1978, } \\
\text { Grohmann et al. 1997, Migotto et al. } 2001\end{array}$ & $\mathrm{P} / \mathrm{M}$ \\
\hline Dipurena sp. & SE & SP & Tronolone 2001 & $\mathrm{M}$ \\
\hline $\begin{array}{l}\text { Nannocoryne mammylia } \text { Bouillon \& Grohmann, } \\
1994\end{array}$ & SE & RJ & Bouillon \& Grohmann 1994 & $\mathrm{P}$ \\
\hline Sarsia eximia Allman, 1859 & SE & SP & Vannucci 1957a & $\mathrm{M}$ \\
\hline Sarsia japonica (Nagao, 1962) & SE & SP & Migotto 1996 & $\mathrm{P} / \mathrm{M}$ \\
\hline Sarsia producta (Wright, 1858) & SE & SP & Vannucci 1949 & $\mathrm{P}$ \\
\hline \multicolumn{5}{|l|}{ Family Eleutheriidae } \\
\hline Staurocladia sp. & SE & ES & Silveira et al. 1988 & $\mathrm{M}$ \\
\hline \multicolumn{5}{|l|}{ Family Euphysidae } \\
\hline $\begin{array}{l}\text { Pinushydra chiquitita Bouillon \& Grohmann, } \\
1990\end{array}$ & SE & RJ & Bouillon \& Grohmann 1990 & $\mathrm{P}$ \\
\hline \multicolumn{5}{|l|}{ Family Milleporidae } \\
\hline Millepora alcicornis Linnaeus, 1758 & SE, NE & $\begin{array}{l}\text { RJ - PI, FN, } \\
\text { AR }\end{array}$ & $\begin{array}{l}\text { Hartt, 1869, Branner, 1904, Laborel } \\
\text { 1969a,b, Eston et al. 1986, Pires et al. } \\
\text { 1992, Hetzel \& Castro 1994, Castro et al. } \\
\text { 1995, Amaral 1997, Amaral et al. 1997, } \\
\text { Echeverría et al. 1997, Calder \& Maÿal } \\
\text { 1998, Kelmo \& de Santa-Isabel 1998, } \\
\text { Castro \& Segal, 2001, Castro \& Pires, } \\
2001\end{array}$ & $\mathrm{P}$ \\
\hline
\end{tabular}




\begin{tabular}{|c|c|c|c|c|}
\hline Millepora braziliensis Verrill, 1868 & SE, NE & ES - RN, AR & $\begin{array}{l}\text { Laborel 1969a b, Hetzel \& Castro 1994, } \\
\text { Amaral 1997, Amaral et al. 1997, } \\
\text { Echeverría et al. 1997, Hudson et al. 1999, } \\
\text { Castro \& Segal, 2001, Castro \& Pires, } \\
2001\end{array}$ & $\mathrm{P}$ \\
\hline Millepora nitida Verrill, 1868 & $\mathrm{NE}$ & $\mathrm{BA}, \mathrm{AL}$ & $\begin{array}{l}\text { Verrill 1868, Laborel 1969a, Hetzel \& } \\
\text { Castro 1994, Amaral 1997, Castro \& } \\
\text { Segal, 2001, Castro \& Pires, } 2001\end{array}$ & $\mathrm{P}$ \\
\hline Millepora sp.1 & NE & BA, PA & Amaral 1997 & $\mathrm{P}$ \\
\hline Millepora $\mathrm{sp} .2$ & $\mathrm{NE}$ & MA & $\begin{array}{l}\text { Amaral 1997, Moura et al. 1999, Castro \& } \\
\text { Pires, } 2001\end{array}$ & $\mathrm{P}$ \\
\hline Millepora squarrosa Lamarck, 1816 & $\mathrm{NE}$ & PE, BA & \begin{tabular}{|l|} 
Laborel 1969b, Weerdt 1984, Amaral \\
1997, Castro \& Pires, 2001 \\
\end{tabular} & $\mathrm{P}$ \\
\hline \multicolumn{5}{|l|}{ Family Moerisiidae } \\
\hline $\begin{array}{l}\text { Moerisia inkermanica Paltschikowa- } \\
\text { Ostroumova, } 1925\end{array}$ & $\mathrm{NE}$ & $\mathrm{PE}$ & $\begin{array}{l}\text { Paranaguá 1963, Ramirez \& Zamponi } \\
1981\end{array}$ & M \\
\hline \multicolumn{5}{|l|}{ Family Pennariidae } \\
\hline Pennaria disticha Goldfuss, 1820 & $\mathrm{~S}, \mathrm{SE}, \mathrm{NE}$ & $\mathrm{SC}-\mathrm{BA}, \mathrm{FN}$ & $\begin{array}{l}\text { Silveira \& Migotto 1991, Migotto 1996, } \\
\text { Grohmann et al. 1997, Rosso \& Marques } \\
\text { 1997, Calder \& Maÿal 1998, Kelmo \& de } \\
\text { Santa-Isabel } 1998\end{array}$ & $\mathrm{P}$ \\
\hline \multicolumn{5}{|l|}{ Family Porpitidae } \\
\hline Porpita umbella F. Müller, 1776 & SE, NE & SP, BA & $\begin{array}{l}\text { Alvariño 1971; Kelmo \& de Santa-Isabel } \\
1998\end{array}$ & $\mathrm{P}$ \\
\hline Velella velella Linnaeus, 1758 & SE, NE & SP, FN & Alvariño 1971 & $\mathrm{P}$ \\
\hline \multicolumn{5}{|l|}{ Family Solanderiidae } \\
\hline $\begin{array}{l}\text { Solanderia gracilis Duchassaing \& Michelin, } \\
1846\end{array}$ & $\mathrm{NE}$ & $\mathrm{BA}$ & Belém et al. 1982 & $\mathrm{P}$ \\
\hline \multicolumn{5}{|l|}{ Family Sphaerocorynidae } \\
\hline Sphaerocoryne arcuata (Haeckel, 1879) & $\begin{array}{l}\text { no specific } \\
\text { record }\end{array}$ & $\begin{array}{l}\text { no specific } \\
\text { record }\end{array}$ & Vannucci 1951b & M \\
\hline Sphaerocoryne sp. & SE & ES & Grohmann et al. 1997 & $\mathrm{P}$ \\
\hline \multicolumn{5}{|l|}{ Family Tubulariidae } \\
\hline Ectopleura dumortierii (Van Beneden, 1844) & S, SE & $\mathrm{RS}-\mathrm{RJ}$ & $\begin{array}{l}\text { Vannucci 1957a 1963, Moreira 1973, } \\
\text { Navas-Pereira } 1980 \text { 1981, Migotto \& } \\
\text { Silveira 1987, Migotto 1996, Tronolone } \\
2001\end{array}$ & $\mathrm{P} / \mathrm{M}$ \\
\hline Ectopleura obypa Migotto \& Marques, 1999 & SE & SP & Migotto \& Marques 1999a & $\mathrm{P} / \mathrm{M}$ \\
\hline Pinauay ralphi (Bale, 1884) & S, SE & RS - ES & $\begin{array}{l}\text { Migotto \& Silveira 1987, Haddad 1992, } \\
\text { Migotto 1996, Grohmann et al. 1997, } \\
\text { Rosso \& Marques 1997, Migotto et al. } \\
2001\end{array}$ & $\mathrm{P}$ \\
\hline $\begin{array}{l}\text { Ralpharia sanctisebastiani (Silveira \& Migotto, } \\
\text { 1984) }\end{array}$ & SE & SP & $\begin{array}{l}\text { Silveira \& Migotto 1984, Migotto \& } \\
\text { Silveira } 1987\end{array}$ & $\mathrm{P}$ \\
\hline Tubularia formosa Hartlaub, 1905 & SE & RJ & ?Stechow 1912 & $\mathrm{P}$ \\
\hline Zyzzyzus warreni Calder, 1988 & SE, NE & $\mathrm{SP}, \mathrm{PE}$ & $\begin{array}{l}\text { Migotto \& Silveira 1987, Migotto 1996, } \\
\text { Calder \& Maÿal 1998, Migotto et al. } 2001\end{array}$ & $\mathrm{P}$ \\
\hline \multicolumn{5}{|l|}{ Family Zancleidae } \\
\hline Zanclea costata Gegenbaur, 1856 & $\mathrm{~S}, \mathrm{SE}$ & RS, SP - ES & $\begin{array}{l}\text { Vannucci 1957a, Navas-Pereira } 1981 \\
\text { 1984a, Migotto } 1996\end{array}$ & $\mathrm{P} / \mathrm{M}$ \\
\hline Zanclea sp. & SE & ES & Grohmann et al. 1997 & $\mathrm{P}$ \\
\hline \multicolumn{5}{|l|}{ Family Zancleopsidae } \\
\hline Zancleopsis dichotoma (Mayer, 1900) & SE & $\mathrm{RJ}$ & Navas-Pereira 1980 & $\mathrm{M}$ \\
\hline \multicolumn{5}{|l|}{ Order Filifera } \\
\hline \multicolumn{5}{|l|}{ Family Bougainvilliidae } \\
\hline Bimeria sp. & SE & SP & Rosso \& Marques 1997 & $\mathrm{P}$ \\
\hline
\end{tabular}




\begin{tabular}{|c|c|c|c|c|}
\hline Bimeria vestita Wright, 1859 & SE, NE & $\mathrm{SP}, \mathrm{ES}, \mathrm{BA}$ & $\begin{array}{l}\text { Migotto 1996, Grohmann et al. 1997, } \\
\text { Rosso \& Marques 1997, Kelmo \& de } \\
\text { Santa-Isabel } 1998\end{array}$ & $\mathrm{P}$ \\
\hline Bougainvillia carolinensis (McCrady, 1859) & SE & SP & $\begin{array}{l}\text { Vannucci 1951a, Vannucci \& Rees 1961, } \\
\text { Tronolone } 2001\end{array}$ & $\mathrm{M}$ \\
\hline Bougainvillia frondosa Mayer, 1900 & S, SE, NE & $\begin{array}{l}\mathrm{RS}, \mathrm{SC}, \mathrm{SP},(\mathrm{no} \\
\text { specific record } \\
\text { for } \mathrm{NE} \text { region) }\end{array}$ & $\begin{array}{l}\text { Moreira 1973, Goy 1979, Navas-Pereira } \\
1981\end{array}$ & $\mathrm{M}$ \\
\hline Bougainvillia muscus (Allman, 1863) & S, SE, NE & $\begin{array}{l}\mathrm{PR}-\mathrm{RJ}, \text { (no } \\
\text { specific record } \\
\text { for NE region) }\end{array}$ & $\begin{array}{l}\text { Vannucci 1957a 1963, Vannucci \& Rees } \\
\text { 1961, Moreira 1973, Goy 1979, Navas- } \\
\text { Pereira 1980, Haddad } 1992\end{array}$ & $\mathrm{M}$ \\
\hline Bougainvillia sp. & SE & $\mathrm{SP}$ & $\begin{array}{l}\text { Rosso \& Marques 1997, Migotto et al. } \\
2001\end{array}$ & $\mathrm{P}$ \\
\hline Bougainvillia trinema (von Lendenfeld, 1884) & $\mathrm{SE}$ & $\mathrm{SC}$ & Correia 1985 & M \\
\hline Garveia franciscana (Torrey, 1902) & $\mathrm{NE}$ & $\mathrm{PE}$ & Calder \& Maÿal 1998 & $\mathrm{P}$ \\
\hline Parawrightia robusta Warren, 1907 & SE, NE & SP, ES, BA & $\begin{array}{l}\text { Grohmann et al.1997, Kelmo \& de Santa- } \\
\text { Isabel 1998, Migotto (unpublished data) }\end{array}$ & $\mathrm{P}$ \\
\hline Rhizorhagium sp. & $S$ & PR & Haddad 1992 & $\mathrm{P}$ \\
\hline \multicolumn{5}{|l|}{ Family Clavidae } \\
\hline Clavidae sp. indet. 1 & $\mathrm{SE}$ & SP & Migotto et al. 2001 & $\mathrm{P}$ \\
\hline Cordylophora caspia (Pallas, 1771) & $\mathrm{S}, \mathrm{SE}$ & $\mathrm{PR}, \mathrm{SP}$ & $\begin{array}{l}\text { Haddad \& Nakatani 1996, Silveira \& } \\
\text { Boscolo } 1996\end{array}$ & $\mathrm{P}$ \\
\hline Corydendrium parasiticum (Linnaeus, 1767) & SE, NE & $\mathrm{SP}, \mathrm{PE}$ & Migotto 1996, Calder \& Maÿal 1998 & $\mathrm{P}$ \\
\hline Rhizodendrium sterreri Calder, 1988 & $\mathrm{NE}$ & BA & Kelmo \& de Santa-Isabel 1998 & $\mathrm{P}$ \\
\hline Tubiclava $\mathrm{sp}$. & SE & RJ & Nogueira et al. 1997 & $\mathrm{P}$ \\
\hline Turritopsis nutricula McCrady, 1857 & S, SE, NE & $\begin{array}{l}\text { RS, SP - BA, } \\
\text { PE }\end{array}$ & $\begin{array}{l}\text { Vannucci 1963, Moreira 1973, Goy 1979, } \\
\text { Navas-Pereira 1981, Migotto 1996, } \\
\text { Grohmann et al. 1997, Rosso \& Marques } \\
\text { 1997, Calder \& Maÿal 1998, Kelmo \& de } \\
\text { Santa-Isabel 1998, Migotto et al. 2001, } \\
\text { Tronolone } 2001\end{array}$ & $\mathrm{P} / \mathrm{M}$ \\
\hline \multicolumn{5}{|l|}{ Family Cytaeididae } \\
\hline Cytaeis tetrastyla Eschscholtz, 1829 & S, SE, NE & RS, SP, PE & $\begin{array}{l}\text { Kramp 1957, Vannucci 1957a 1963, } \\
\text { Moreira 1973, Goy 1979, Navas-Pereira } \\
\text { 1981, Tronolone } 2001\end{array}$ & $\mathrm{M}$ \\
\hline
\end{tabular}




\begin{tabular}{|c|c|c|c|c|}
\hline Eudendrium carneum Clarke, 1882 & S, SE, NE & $\begin{array}{l}\text { SC, SP - BA, } \\
\text { PE, FN }\end{array}$ & $\begin{array}{l}\text { Vannucci 1954, Eston et al. 1986, Pires et } \\
\text { al. 1992, Marques } 1993 \text { 2001, Marques \& } \\
\text { Moretzsohn 1995, Grohmann et al. 1997, } \\
\text { Nogueira et al. 1997, Rosso \& Marques } \\
\text { 1997, Calder \& Maÿal 1998, Kelmo \& de } \\
\text { Santa-Isabel 1998, Oliveira 2000, Oliveira } \\
\text { et al. } 2000\end{array}$ & $\mathrm{P}$ \\
\hline Eudendrium fragile Motz-Kossowska, 1905 & SE & ES & ?Grohmann et al. 1997 & $\mathrm{P}$ \\
\hline Eudendrium glomeratum Picard, 1951 & $\mathrm{SE}$ & SP & $\begin{array}{l}\text { Marques } 1993 \text { 2001, Rosso \& Marques } \\
\text { 1997, Oliveira 2000, Oliveira et al. 2000, } \\
\text { Migotto et al. } 2001\end{array}$ & $\mathrm{P}$ \\
\hline Eudendrium merulum Watson, 1985 & $\mathrm{~S}$ & $\mathrm{SC}$ & ?Marques 19932001 & $\mathrm{P}$ \\
\hline Eudendrium nambuccense Watson, 1985 & SE & RJ & Marques 1993 2001, Nogueira et al. 1997 & $\mathrm{P}$ \\
\hline Eudendrium pocaruquarum Marques, 1995 & SE & SP & $\begin{array}{l}\text { Marques } 1995 \text { 2001, Rosso \& Marques } \\
1997\end{array}$ & $\mathrm{P}$ \\
\hline Eudendrium ramosum (Linnaeus, 1758) & SE & SP, ES & $\begin{array}{l}\text { Marques } 19931995 \text { 2001, Grohmann et al. } \\
\text { 1997, Rosso \& Marques } 1997 \\
\end{array}$ & $\mathrm{P}$ \\
\hline Eudendrium sp. & $\mathrm{S}, \mathrm{SE}$ & $\mathrm{RS}, \mathrm{RJ}$ & Nogueira et al. 1997, Horta et al. 2001 & $\mathrm{P}$ \\
\hline \multicolumn{5}{|l|}{ Family Hydractiniidae } \\
\hline Hydractinia minima (Trinci, 1903) & SE & SP, RJ & $\begin{array}{l}\text { Vannucci 1957a 1963, Navas-Pereira } \\
\text { 1980, Tronolone } 2001\end{array}$ & $\mathrm{M}$ \\
\hline Hydractinia minuta (Mayer, 1900) & SE & SP & Tronolone 2001 & $\mathrm{M}$ \\
\hline Stylactaria hooperii (Sigerfoos, 1899) & SE & SP & Moreira et al. 1979, Migotto 1996 & $\mathrm{P} / \mathrm{M}$ \\
\hline Stylactaria sp. & SE, NE & SP, PE & Migotto 1996, Calder \& Maÿal 1998 & $\mathrm{P}$ \\
\hline \multicolumn{5}{|l|}{ Family Niobiidae } \\
\hline Niobia dendrotentaculata Mayer, 1900 & $S$ & RS & Goy 1979 & $\mathrm{M}$ \\
\hline \multicolumn{5}{|l|}{ Family Pandeidae } \\
\hline Amphinema australis (Mayer, 1900) & SE & RJ & Navas-Pereira 1980 & $\mathrm{M}$ \\
\hline Amphinema dinema (Péron \& Lesueur, 1809) & $\mathrm{S}, \mathrm{SE}$ & RS, SP, ES & $\begin{array}{l}\text { Vannucci 1957a 1963, Goy 1979, Navas- } \\
\text { Pereira 1981, Grohmann et al. } 1997\end{array}$ & $\mathrm{P} / \mathrm{M}$ \\
\hline Amphinema rugosum (Mayer, 1900) & SE & SP & Migotto 1996 & $\mathrm{P} / \mathrm{M}$ \\
\hline Amphinema sp. & SE & SP & Tronolone 2001 & $\mathrm{M}$ \\
\hline Annatiara affinis (Hartlaub, 1913) & S & RS & Navas-Pereira 1981 & $\mathrm{M}$ \\
\hline Cirrhitiara superba (Mayer, 1900) & $\mathrm{S}, \mathrm{NE}$ & $\begin{array}{l}\text { RS, (no } \\
\text { specific record } \\
\text { for NE region) }\end{array}$ & $\begin{array}{l}\text { Thiel 1938, Navas-Pereira 1981, Ramirez } \\
\& \text { Zamponi } 1981\end{array}$ & $\mathrm{M}$ \\
\hline Leuckartiara octona (Fleming, 1823) & $\mathrm{S}, \mathrm{SE}$ & RS, SP, ES & \begin{tabular}{|l|} 
Navas-Pereira 1981, Migotto 1996, \\
Grohmann et al. 1997, Tronolone 2001 \\
\end{tabular} & $\mathrm{P} / \mathrm{M}$ \\
\hline Merga tergestina (Neppi \& Stiasny, 1911) & $\mathrm{S}, \mathrm{SE}$ & RS, SP & Moreira 1973, Navas-Pereira 1981 & $\mathrm{M}$ \\
\hline Merga violacea (Agassiz \& Mayer, 1899) & $\mathrm{NE}$ & BA & Goy 1979 & $\mathrm{M}$ \\
\hline Pandea conica (Quoy \& Gaimard, 1827) & $\mathrm{S}, \mathrm{SE}, \mathrm{NE}$ & $\begin{array}{l}\text { no specific } \\
\text { record }\end{array}$ & Ramirez \& Zamponi 1981 & $\mathrm{M}$ \\
\hline \multicolumn{5}{|l|}{ Family Proboscidactylidae } \\
\hline Proboscidactyla ornata (McCrady, 1859) & $\mathrm{S}, \mathrm{SE}, \mathrm{NE}$ & RS - RJ, AL & $\begin{array}{l}\text { Vannucci 1957a 1963, Moreira 1973, } \\
\text { Navas-Pereira 1981, Tronolone } 2001\end{array}$ & $\mathrm{M}$ \\
\hline Proboscidactyla $\mathrm{sp.}$ & SE & SP & Tronolone 2001 & $\mathrm{M}$ \\
\hline \multicolumn{5}{|l|}{ Family Protiaridae } \\
\hline Halitiara formosa Fewkes, 1882 & SE & SP & Tronolone 2001 & $\mathrm{M}$ \\
\hline \multicolumn{5}{|l|}{ Family Rhysiidae } \\
\hline Rhysia sp. & SE & ES & Grohmann et al. 1997 & $\mathrm{P}$ \\
\hline \multicolumn{5}{|l|}{ Family Stylasteridae } \\
\hline Stylaster roseus (Pallas, 1766) & $\mathrm{NE}$ & PE, FN, MA & $\begin{array}{l}\text { Laborel 1969a b, Pires et al. 1992, Hudson } \\
\text { et al. } 1999\end{array}$ & $\mathrm{P}$ \\
\hline
\end{tabular}




\begin{tabular}{|c|c|c|c|c|}
\hline Subclass Leptomedusae & & & & \\
\hline Order Conica & & & & \\
\hline Family Aequoreidae & & & & \\
\hline Aequorea forskalea Péron \& Lesueur, 1810 & $\mathrm{~S}, \mathrm{NE}$ & RS, BA, PE & Goy 1979, Navas-Pereira 1981 & $\mathrm{M}$ \\
\hline Rhacostoma atlantica L. Agassiz, 1850 & S, SE & RS, SP & $\begin{array}{l}\text { Moreira 1973, Goy 1979, Navas-Pereira } \\
\text { 1981, Tronolone } 2001\end{array}$ & $\mathrm{M}$ \\
\hline Aequoridae sp. indet. 1 & SE & SP & Migotto et al. 2001, Tronolone 2001 & $\mathrm{P} / \mathrm{M}$ \\
\hline Family Aglaopheniidae & & & & \\
\hline Aglaophenia calamus Allman, 1883 & NE & $\mathrm{BA}$ & ?Allman 1883 & $\mathrm{P}$ \\
\hline Aglaophenia dubia Nutting, 1900 & $\mathrm{NE}$ & $\mathrm{BA}$ & Ritchie 1909 & $\mathrm{P}$ \\
\hline Aglaophenia insignis Fewkes, 1881 & $\mathrm{NE}$ & $\mathrm{PE}$ & Maÿal 1983 & $\mathrm{P}$ \\
\hline Aglaophenia latecarinata Allman, 1887 & S, SE, NE & SC - BA, FN & $\begin{array}{l}\text { Nutting 1900, Ritchie 1909, Vervoort } \\
\text { 1946, Vannucci Mendes 1946, Vannucci } \\
\text { 1949 1951a, Maÿal 1983, Pires et al. 1992, } \\
\text { Migotto 1996, Grohmann et al. 1997, } \\
\text { Nogueira et al. 1997, Rosso \& Marques } \\
\text { 1997, Haddad et al. 2000, Andrade } 2001\end{array}$ & $\mathrm{P}$ \\
\hline Aglaophenia latirostris Nutting, 1900 & BR & $\begin{array}{l}\text { no specific } \\
\text { record }\end{array}$ & ?Nutting 1900 & $\mathrm{P}$ \\
\hline Aglaophenia perforata Allman, 1885 & SE & ES & Vannucci 1951a & $\mathrm{P}$ \\
\hline Aglaophenia rhynchocarpa Allman, 1877 & $\mathrm{NE}$ & $\mathrm{BA}$ & Nutting 1900 & $\mathrm{P}$ \\
\hline Aglaophenia rigida Allman, 1887 & SE & SP, RJ & Vannucci 19501954 & $\mathrm{P}$ \\
\hline $\begin{array}{l}\text { Gymnangium allmani (Marktanner- } \\
\text { Turneretscher, 1890) }\end{array}$ & SE & RJ & Nogueira et al. 1997 & $\mathrm{P}$ \\
\hline Gymnangium longicaudum (Nutting, 1900) & $\mathrm{NE}$ & BA & Ritchie 1909 & $\mathrm{P}$ \\
\hline Lytocarpia tridentata (Versluys, 1889) & SE, NE & SP, RJ, PE & $\begin{array}{l}\text { Totton 1926, Vannucci Mendes 1946, } \\
\text { Vannucci 1951a, Migotto 1996, Rosso \& } \\
\text { Marques } 1997\end{array}$ & $\mathrm{P}$ \\
\hline Macrorhynchia allmani (Nutting, 1900) & $\mathrm{NE}$ & $\mathrm{BA}$ & Ritchie 1909 & $\mathrm{P}$ \\
\hline $\begin{array}{l}\text { Macrorhynchia philippina (Kirchenpauer, } \\
\text { 1872) }\end{array}$ & SE, NE & SP, RJ, BA, PE & $\begin{array}{l}\text { Nutting 1900, Vannucci Mendes 1946, } \\
\text { Vannucci 1949 1954, Migotto 1996, } \\
\text { Grohmann et al. 1997, Nogueira et al. } \\
\text { 1997, Calder \& Maÿal 1998 }\end{array}$ & $\mathrm{P}$ \\
\hline Macrorhynchia racemifera (Allman, 1883) & $\mathrm{NE}$ & $\mathrm{BA}$ & $\begin{array}{l}\text { Allman 1883, Marques \& Moretzsohn } \\
1995\end{array}$ & $\mathrm{P}$ \\
\hline Thecocarpus laxus Billard, 1913 & SE & $\mathrm{RJ}$ & ?Vannucci 1950 & $\mathrm{P}$ \\
\hline Family Blackfordiidae & & & & \\
\hline Blackfordia virginica Mayer, 1910 & $\mathrm{NE}$ & $\mathrm{PE}$ & Paranaguá 1963 & $\mathrm{M}$ \\
\hline Family Campanulinidae & & & & \\
\hline Calycella gabriellae Vannucci, 1951a & SE & SP, ES & Vannucci 1951a, Grohmann et al. 1997 & $\mathrm{P}$ \\
\hline Campanulinidae sp. indet. 1 & SE & SP & Migotto et al. 2001 & $\mathrm{P}$ \\
\hline Cuspidella humilis (Hincks, 1868) & SE & $\mathrm{RJ}$ & Vannucci 1949 & $\mathrm{P}$ \\
\hline Cuspidella sp. & SE & SP & Migotto et al. 2001 & \\
\hline $\begin{array}{l}\text { Lafoeina amirantensis Millard \& Bouillon, } \\
1973\end{array}$ & SE, NE & RJ, PE & $\begin{array}{l}\text { Nogueira et al. 1997, Calder \& Maÿal } \\
1998\end{array}$ & $\mathrm{P}$ \\
\hline Tetracanna octonema Goy, 1979 & $\mathrm{NE}$ & $\mathrm{AL}$ & Goy 1979 & $\mathrm{M}$ \\
\hline Family Cirrholoveniidae & & & & \\
\hline Cirrholovenia tetranema Kramp, 1959 & S, SE, NE & $\begin{array}{l}\text { RS, SP (no } \\
\text { specific record } \\
\text { for NE region) }\end{array}$ & $\begin{array}{l}\text { Moreira 1975, Goy 1979, Navas-Pereira } \\
\text { 1981, Tronolone } 2001\end{array}$ & $\mathrm{M}$ \\
\hline Family Dipleurosomatidae & & & & \\
\hline Dipleurosoma collapsum (Mayer, 1900) & $\mathrm{NE}$ & $\mathrm{PE}$ & Goy 1979 & $\mathrm{M}$ \\
\hline Family Eirenidae & & & & \\
\hline Eirene viridula (Péron \& Lesueur, 1809) & $\mathrm{N}$ & $\mathrm{PA}$ & Alvariño 1968 & $\mathrm{M}$ \\
\hline \begin{tabular}{|l|} 
Eutima coerulea $($ L. Agassiz, 1862) \\
\end{tabular} & $S$ & RS & Navas-Pereira 1981 & $\mathrm{M}$ \\
\hline
\end{tabular}




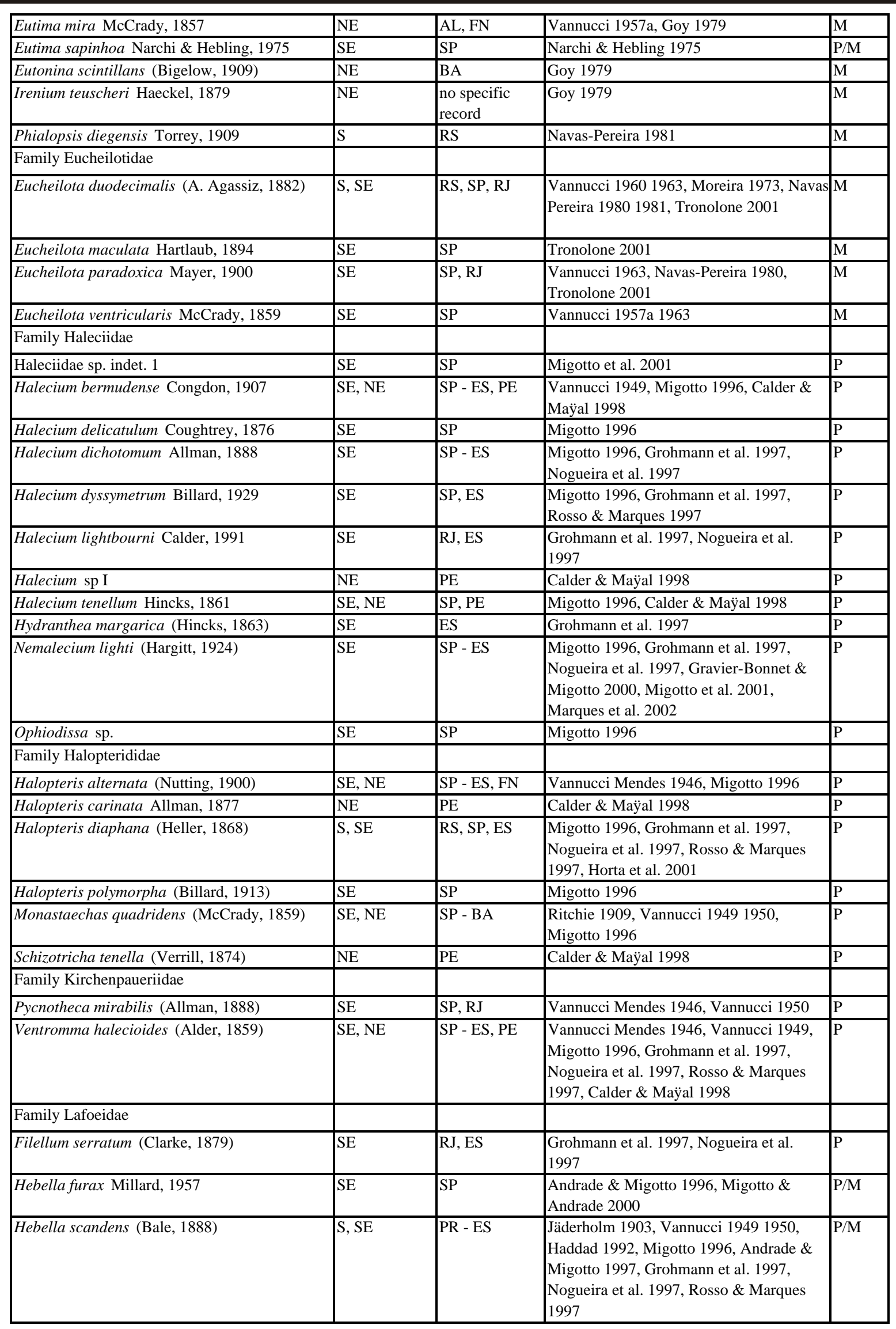




\begin{tabular}{|c|c|c|c|c|}
\hline Hebella sp. & SE & ES & Grohmann et al. 1997 & $\mathrm{P}$ \\
\hline Hebellopsis communis Calder, 1991 & SE & SP - ES & ?Vannucci 19491950 1951a 1954 & $\mathrm{P}$ \\
\hline Scandia mutabilis (Ritchie, 1907) & SE & SP & Migotto 1996 & $\mathrm{P}$ \\
\hline \multicolumn{5}{|l|}{ Family Laodiceidae } \\
\hline Laodicea minuscula Vannucci, 1957 & SE & SP, RJ & Vannucci 1957a 1963, Moreira 1973 & $\mathrm{M}$ \\
\hline Laodicea undulata (Forbes \& Goodsir, 1851) & S, NE & $\mathrm{RS}, \mathrm{BA}, \mathrm{PE}$ & $\begin{array}{l}\text { Kramp 1957, Goy 1979, Navas-Pereira } \\
1981\end{array}$ & $\mathrm{M}$ \\
\hline \multicolumn{5}{|l|}{ Family Lovenellidae } \\
\hline Lovenella cirrata (Haeckel, 1879) & SE, $\mathrm{N}$ & $\begin{array}{l}\text { PA (no specific } \\
\text { record for SE } \\
\text { region) }\end{array}$ & Thiel 1938, Vannucci 1957a & $\mathrm{M}$ \\
\hline \multicolumn{5}{|l|}{ Family Malagazziidae } \\
\hline Malagazzia carolinae (Mayer, 1900) & S, SE & RS, RJ & Navas-Pereira 19801981 1984b & $\mathrm{M}$ \\
\hline Malagazziidae sp. indet. 1 & SE & SP & Tronolone 2001 & $\mathrm{M}$ \\
\hline Octacanna haeckeli Vannucci \& Moreira, 1966 & SE & $\mathrm{SP}$ & Vannucci \& Moreira 1966 & $\mathrm{M}$ \\
\hline Octophialucium bigelowi Kramp, 1955 & SE, NE & $\mathrm{SP}, \mathrm{AL}$ & $\begin{array}{l}\text { Vannucci \& Moreira 1966, Goy 1979, } \\
\text { Tronolone } 2001\end{array}$ & $\mathrm{M}$ \\
\hline \multicolumn{5}{|l|}{ Family Mitrocomidae } \\
\hline Cosmetirella davisi (Browne, 1902) & $S$ & RS & Navas-Pereira 1981 & $\mathrm{M}$ \\
\hline Mitrocomella brownei (Kramp, 1930) & $S$ & RS & Navas-Pereira 1981 & $\mathrm{M}$ \\
\hline \multicolumn{5}{|l|}{ Family Plumulariidae } \\
\hline Dentitheca bidentata Jäderholm, 1920 & SE, NE & $\begin{array}{l}\text { SP - ES, AL, } \\
\text { PE }\end{array}$ & $\begin{array}{l}\text { Jäderholm 1920, Migotto 1997, Grohmann } \\
\text { et al. 1997, Nogueira et al. 1997, Migotto } \\
\text { \& Marques 1999b }\end{array}$ & P \\
\hline Monotheca margaretta Nutting, 1900 & S, SE, NE & SC - ES, PE & $\begin{array}{l}\text { Vannucci Mendes 1946, Vannucci } 1949 \\
1950 \text { 1951a, Haddad 1992, Migotto 1996, } \\
\text { Grohmann et al. 1997, Nogueira et al. } \\
\text { 1997, Rosso \& Marques 1997, Calder \& } \\
\text { Maÿal 1998, Haddad et al. } 2000\end{array}$ & $\mathrm{P}$ \\
\hline Plumularia floridana Nutting, 1900 & SE, NE & SP - ES, PE & $\begin{array}{l}\text { Vannucci Mendes 1946, Vannucci 1949, } \\
\text { Migotto 1996, Grohmann et al. 1997, } \\
\text { Rosso \& Marques 1997, Calder \& Maÿal } \\
1998\end{array}$ & $\mathrm{P}$ \\
\hline Plumularia obliqua (Johnston, 1847) & SE & RJ & Nogueira et al. 1997 & $\mathrm{P}$ \\
\hline Plumularia setacea (Linnaeus, 1758) & $\mathrm{SE}$ & SP - ES & $\begin{array}{l}\text { Vannucci Mendes 1946, Vannucci } 1949 \\
\text { 1950, Grohmann et al. 1997, Nogueira et } \\
\text { al. 1997 }\end{array}$ & $\mathrm{P}$ \\
\hline Plumularia strictocarpa Pictet, 1893 & $\mathrm{SE}$ & SP - ES & $\begin{array}{l}\text { Vannucci 1949, Migotto 1996, Migotto et } \\
\text { al. } 2001\end{array}$ & $\mathrm{P}$ \\
\hline Plumularia strobilophora Billard, 1913 & SE & ES & Vannucci 1951a & $\mathrm{P}$ \\
\hline \multicolumn{5}{|l|}{ Family Sertulariidae } \\
\hline Amphisbetia operculata (Linnaeus, 1758) & SE & RJ & ?Vannucci 1954 & $\mathrm{P}$ \\
\hline Calamphora campanulata (Warren, 1908) & SE & ES & Grohmann et al. 1997 & $\mathrm{P}$ \\
\hline Diphasia digitalis (Busk, 1852) & SE, NE & SP, ES, BA, PE & $\begin{array}{l}\text { Allman 1888, Vannucci 1949, Maÿal } \\
\text { 1983, Calder \& Maÿal } 1998 \\
\end{array}$ & $\mathrm{P}$ \\
\hline Diphasia tropica Nutting, 1904 & $\mathrm{SE}$ & SP - ES & $\begin{array}{l}\text { Vannucci 1949, Migotto 1996, Grohmann } \\
\text { et al. 1997, Nogueira et al. } 1997\end{array}$ & $\mathrm{P}$ \\
\hline Dynamena crisioides Lamouroux, 1824 & SE, NE & $\begin{array}{l}\text { SP - BA, PE, } \\
\text { FN }\end{array}$ & $\begin{array}{l}\text { Nutting 1904, Vannucci Mendes 1946, } \\
\text { Vannucci 1949 1954, Haddad 1992, Pires } \\
\text { et al. 1992, Migotto 1996, Grohmann et al. } \\
\text { 1997, Rosso \& Marques 1997, Calder \& } \\
\text { Maÿal } 1998\end{array}$ & P \\
\hline Dynamena dalmasi (Versluys, 1899) & SE, NE & SP, BA & Ritchie 1909, Migotto 1996 & \\
\hline
\end{tabular}




\begin{tabular}{|c|c|c|c|c|}
\hline Dynamena disticha (Bosc, 1802) & $\mathrm{S}, \mathrm{SE}, \mathrm{NE}$ & $\begin{array}{l}\text { RS - BA, PE, } \\
\text { FN }\end{array}$ & $\begin{array}{l}\text { Ritchie 1909, Vannucci Mendes 1946, } \\
\text { Vannucci 1949 1950 1951a, Pires et al. } \\
\text { 1992, Migotto 1996, Grohmann et al. } \\
\text { 1997, Nogueira et al. 1997, Rosso \& } \\
\text { Marques 1997, Calder \& Maÿal 1998, } \\
\text { Haddad et al. 2000, Horta et al. } 2001\end{array}$ & $\mathrm{P}$ \\
\hline $\begin{array}{l}\text { Dynamena quadridentata (Ellis \& Solander, } \\
\text { 1786) }\end{array}$ & SE, NE & SP - BA & $\begin{array}{l}\text { Vannucci Mendes 1946, Vannucci } 1949 \\
1950 \text { 1951a, Migotto 1996, Grohmann et } \\
\text { al. 1997, Nogueira et al. } 1997\end{array}$ & $\mathrm{P}$ \\
\hline 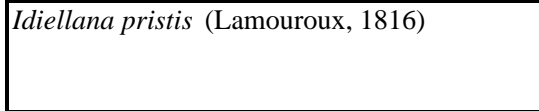 & SE, NE & $\mathrm{SP}, \mathrm{BA}$ & $\begin{array}{l}\text { Allman 1888, Vervoort 1946, Vannucci } \\
\text { Mendes 1946, Migotto 1996, Rosso \& } \\
\text { Marques } 1997\end{array}$ & $\mathrm{P}$ \\
\hline Sertularella areyi Nutting, 1904 & SE & ES & Vannucci 1949 & $P$ \\
\hline Sertularella cylindritheca (Allman, 1888) & SE, NE & $\begin{array}{l}\text { SP, ES, BA, } \\
\text { PE, FN }\end{array}$ & $\begin{array}{l}\text { Allman 1888, Maÿal 1983, Pires et al. } \\
\text { 1992, Migotto 1996, Grohmann et al. } 1997\end{array}$ & $\mathrm{P}$ \\
\hline Sertularella diaphana (Allman, 1885) & $\mathrm{NE}$ & BA, PE, FN & $\begin{array}{l}\text { Allman 1888, Nutting 1904, Vannucci } \\
\text { 1951b, Pires et al. } 1992\end{array}$ & $\mathrm{P}$ \\
\hline $\begin{array}{l}\text { Sertularella moluccana (von Campenhausen, } \\
1896 \text { ) }\end{array}$ & SE & SP & Vannucci Mendes 1946 & $\mathrm{P}$ \\
\hline Sertularia distans (Lamouroux, 1816) & S, SE, NE & $\begin{array}{l}\text { SC - BA, PE, } \\
\text { FN }\end{array}$ & $\begin{array}{l}\text { Ritchie 1909, Vannucci Mendes 1946, } \\
\text { Vannucci 1949 1950, Maÿal 1983, Haddad } \\
\text { 1992, Pires et al. 1992, Migotto 1996, } \\
\text { Grohmann et al. 1997, Nogueira et al. } \\
\text { 1997, Rosso \& Marques 1997, Calder \& } \\
\text { Maÿal 1998, Haddad et al. } 2000\end{array}$ & P \\
\hline Sertularia marginata Kirchenpauer, 1864 & S, SE, NE & $\begin{array}{l}\text { PR - BA, PE, } \\
\text { FN }\end{array}$ & $\begin{array}{l}\text { Allman 1888, Vannucci Mendes 1946, } \\
\text { Vannucci 1949 } 1950 \text { 1951a 1954, Haddad } \\
\text { 1992, Pires et al. 1992, Migotto } 1996 \\
\text { 1998, Grohmann et al. 1997, Nogueira et } \\
\text { al. 1997, Rosso \& Marques } 1997\end{array}$ & $\mathrm{P}$ \\
\hline Sertularia notabilis Fraser, 1947 & SE & $\mathrm{SP}, \mathrm{ES}$ & Migotto \& Vervoort 1998 & $\mathrm{P}$ \\
\hline Sertularia perpusilla Stechow, 1911 & SE & RJ & ?Vannucci 1951a & $\mathrm{P}$ \\
\hline Sertularia rugosissima Thornely, 1904 & S, SE, NE & $\begin{array}{l}\text { PR - ES (no } \\
\text { specific record } \\
\text { for NE region) }\end{array}$ & $\begin{array}{l}\text { Vannucci Mendes 1946, Vannucci 1954, } \\
\text { Migotto 1996, Grohmann et al. 1997, } \\
\text { Nogueira et al. 1997, Rosso \& Marques } \\
1997\end{array}$ & $\mathrm{P}$ \\
\hline Sertularia turbinata (Lamouroux, 1816) & S, SE, NE & PR - ES, PE & $\begin{array}{l}\text { Vannucci Mendes 1946, Vannucci 1949, } \\
\text { Maÿal 1983, Migotto 1996, Grohmann et } \\
\text { al. 1997, Nogueira et al. 1997, Rosso \& } \\
\text { Marques } 1997\end{array}$ & $\mathrm{P}$ \\
\hline Sertularia vervoorti Migotto \& Calder, 1998 & SE & ES & Migotto \& Calder 1998 & $\mathrm{P}$ \\
\hline Thyroscyphus marginatus (Allman, 1877) & $\mathrm{SE}$ & RJ, ES & Vannucci 19491950 1951a, Maÿal 1983 & $\mathrm{P}$ \\
\hline
\end{tabular}




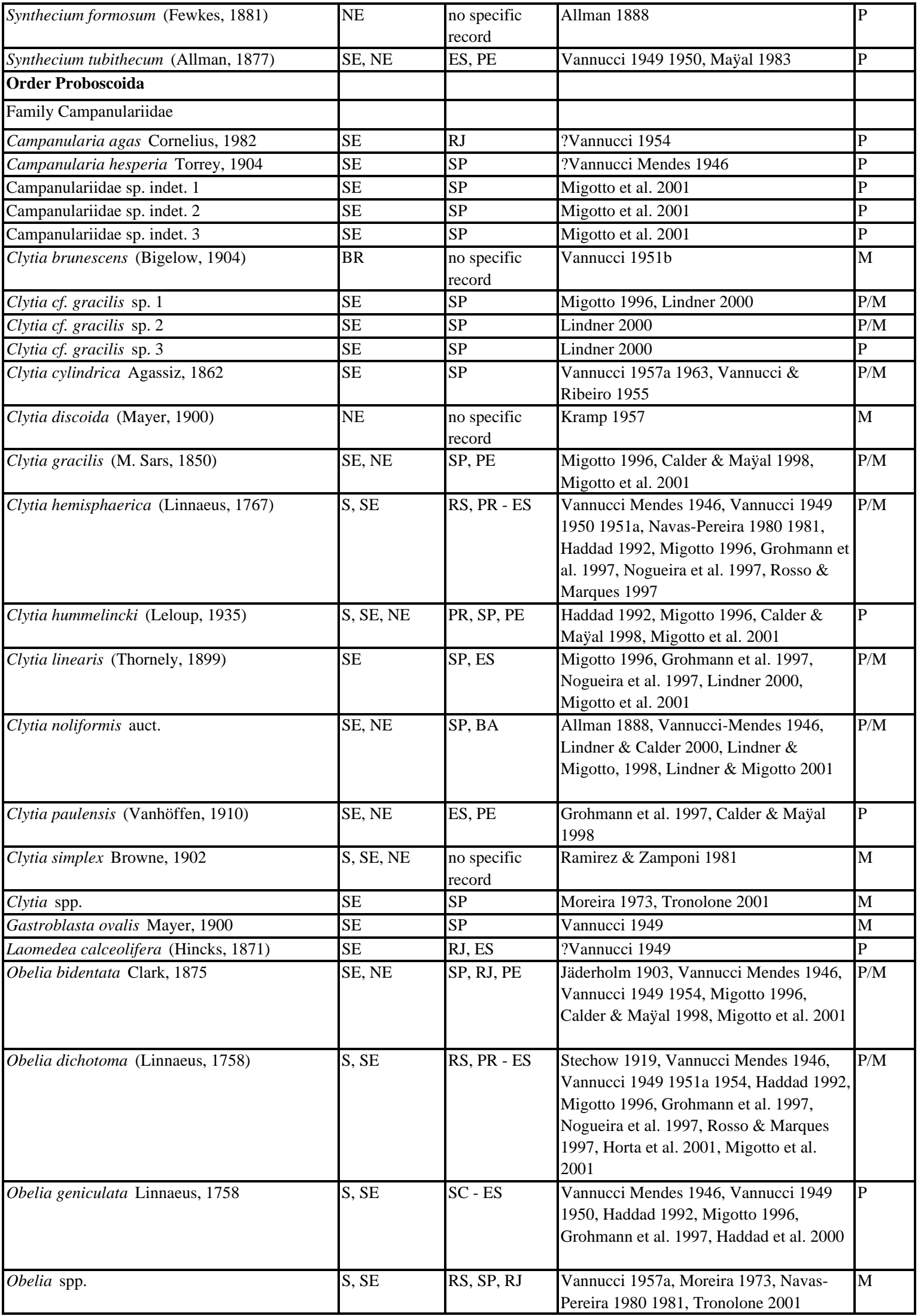




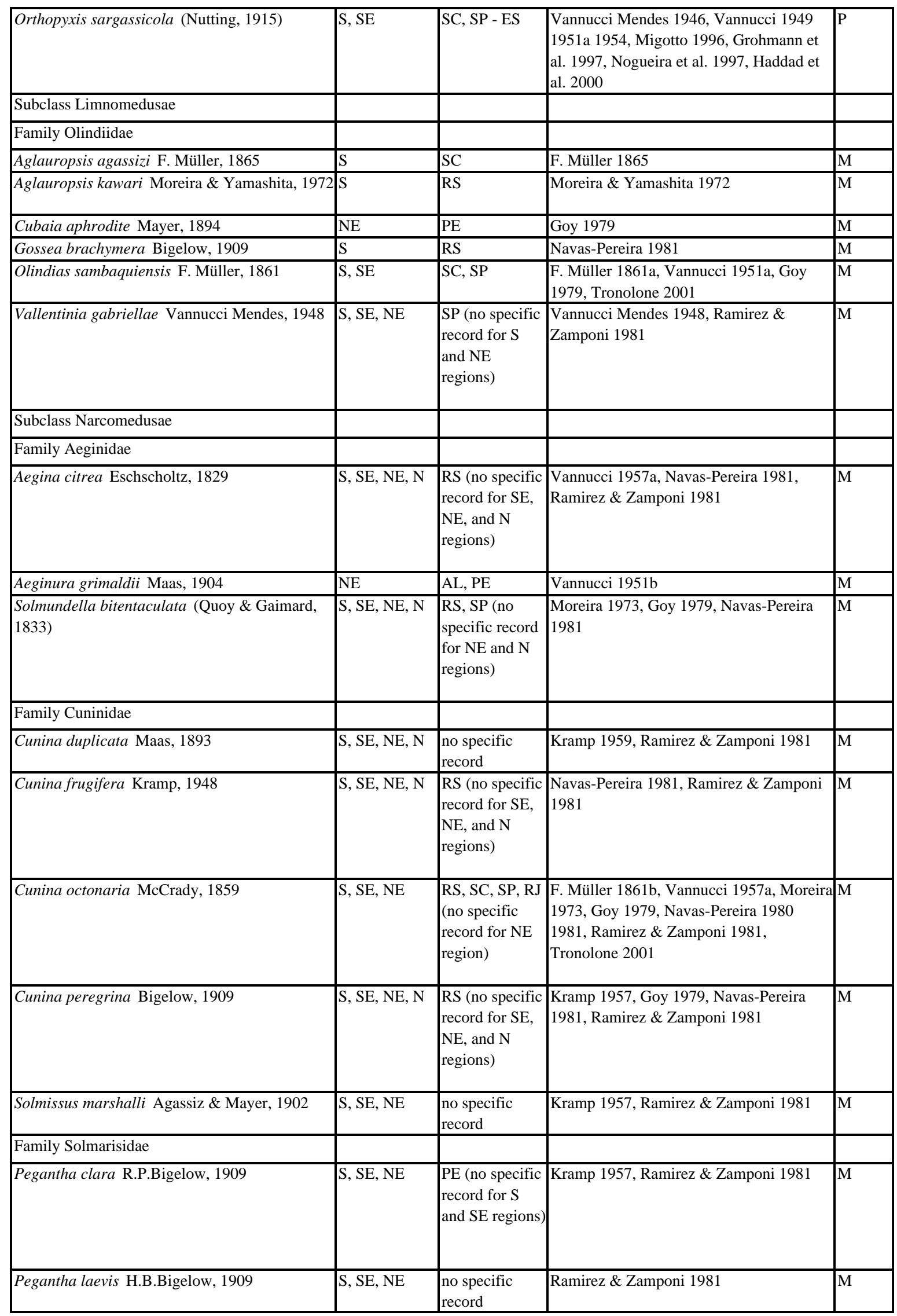




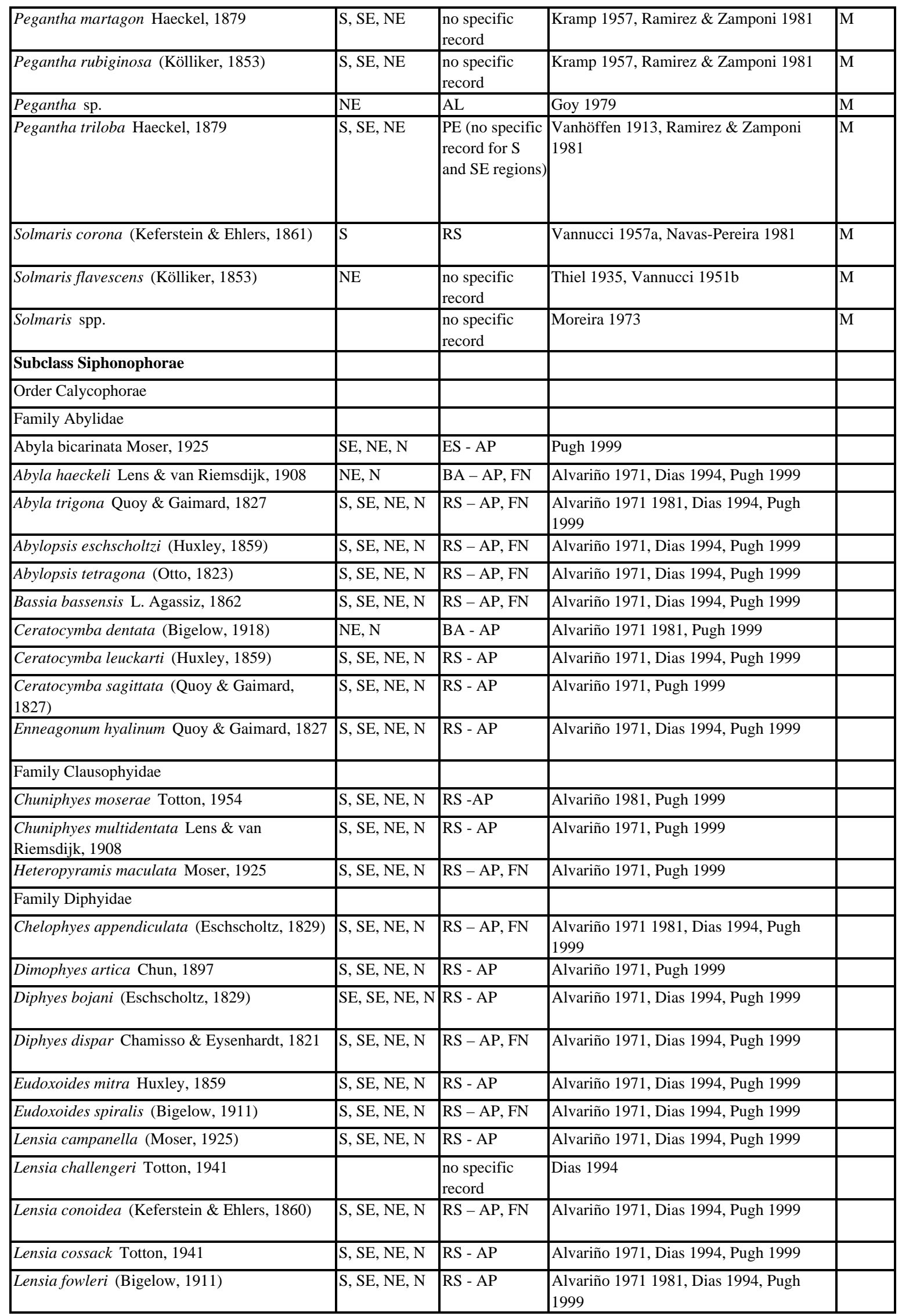




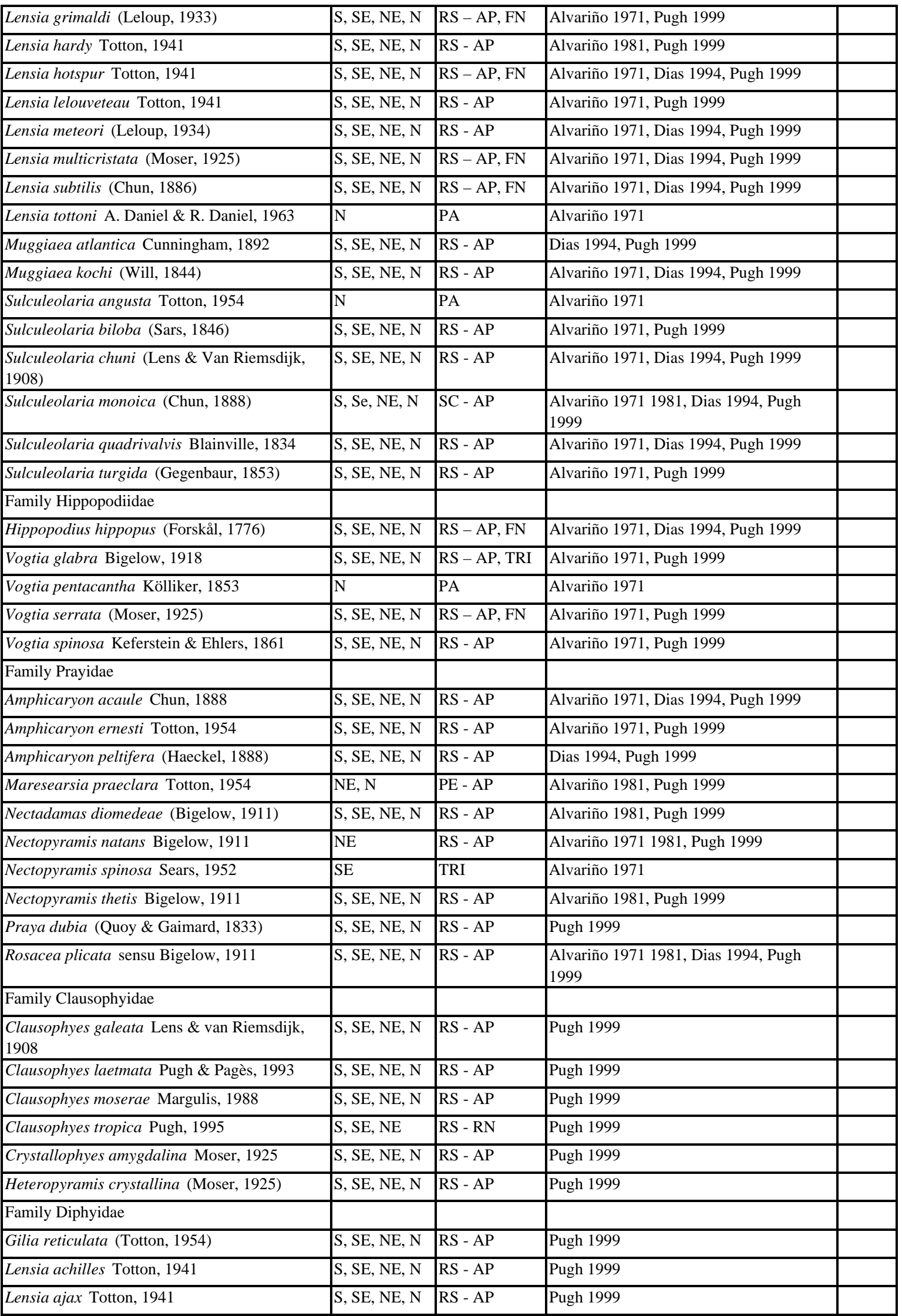




\begin{tabular}{|c|c|c|c|c|}
\hline Lensia exeter Totton, 1941 & $\mathrm{~S}, \mathrm{SE}, \mathrm{NE}, \mathrm{N}$ & RS - AP & Pugh 1999 & \\
\hline Lensia havock Totton, 1941 & $\mathrm{~S}, \mathrm{SE}, \mathrm{NE}, \mathrm{N}$ & RS - AP & Pugh 1999 & \\
\hline Lensia hostile Totton, 1941 & $\mathrm{~S}, \mathrm{SE}, \mathrm{NE}, \mathrm{N}$ & $\mathrm{RS}-\mathrm{AP}$ & Pugh 1999 & \\
\hline Lensia hunter Totton, 1941 & $\mathrm{~S}, \mathrm{SE}, \mathrm{NE}, \mathrm{N}$ & $\mathrm{RS}-\mathrm{AP}$ & Pugh 1999 & \\
\hline Lensia leloupi Totton, 1954 & $\mathrm{NE}, \mathrm{N}$ & MA - AP & Pugh 1999 & \\
\hline Physalia sp. & $\mathrm{N}$ & $\begin{array}{l}\text { No specific } \\
\text { record }\end{array}$ & Hartt, 1868 & \\
\hline Physalia physalis Linnaeus 1758 & S, SE, NE, N & RS - AP & Freitas et al. 1995, Pugh 1999 & \\
\hline Order Physonectae & & & & \\
\hline Agalma okeni Eschscholtz, 1825 & S, SE, NE, N & $\mathrm{RS}-\mathrm{AP}, \mathrm{FN}$ & Alvariño 1971, Dias 1994, Pugh 1999 & \\
\hline Bargmannia elongata Totton, 1954 & S, SE, NE, N & $\mathrm{RS}-\mathrm{AP}$ & Alvariño 1981, Pugh 1999 & \\
\hline Erenna richardi Bedot, 1904 & $\mathrm{~S}, \mathrm{SE}, \mathrm{NE}, \mathrm{N}$ & $\mathrm{RS}-\mathrm{AP}$ & Pugh 1999 & \\
\hline Halistemma rubrum (Vogt, 1852) & $\mathrm{S}, \mathrm{SE}, \mathrm{NE}, \mathrm{N}$ & $\mathrm{RS}-\mathrm{AP}$ & Pugh 1999 & \\
\hline Halistemma striata Totton, 1965 & $\mathrm{~S}, \mathrm{SE}, \mathrm{NE}, \mathrm{N}$ & RS - AP & Pugh 1999 & \\
\hline Marrus antarcticus Totton, 1954 & $\mathrm{~S}$ & $\mathrm{RS}$ & Alvariño 1981 & \\
\hline Marrus orthocanna (Kramp, 1942) & $\mathrm{S}, \mathrm{NE}$ & $\begin{array}{l}\text { no specific } \\
\text { record }\end{array}$ & Alvariño 1981 & \\
\hline Forskalia edwardsii Kölliker, 1853 & $\mathrm{~N}$ & $\mathrm{PA}$ & Alvariño 1971 & \\
\hline Family Physophoridae & & & & \\
\hline Physophora hydrostatica Forskål, 1775 & $\mathrm{~S}, \mathrm{SE}, \mathrm{NE}, \mathrm{N}$ & RS - AP & Dias 1994, Pugh 1999 & \\
\hline Subclass Trachymedusae & & & & \\
\hline Family Geryoniidae & & & & \\
\hline Geryonia proboscidalis (Forskål, 1775) & $\mathrm{S}, \mathrm{NE}$ & RS, FN & $\begin{array}{l}\text { Vannucci 1951b, Goy 1979, Navas-Pereira } \\
1981\end{array}$ & $\mathrm{M}$ \\
\hline $\begin{array}{l}\text { Liriope tetraphylla (Chamisso \& Eysenhardt, } \\
\text { 1821) }\end{array}$ & $\mathrm{S}, \mathrm{SE}$ & $\mathrm{RS}, \mathrm{SC}, \mathrm{SP}, \mathrm{RJ}$ & $\begin{array}{l}\text { F. Müller 1859a, Carvalho 1943, Vannucci } \\
\text { 1951a 1957a, Moreira 1973, Navas-Pereira } \\
1980 \text { 1981, Tronolone } 2001\end{array}$ & M \\
\hline Family Halicreatidae & & & & \\
\hline Halicreas minimum Fewkes, 1882 & $\mathrm{NE}$ & $\begin{array}{l}\text { no specific } \\
\text { record }\end{array}$ & Vannucci 1951b & $\mathrm{M}$ \\
\hline Family Rhopalonematidae & & & & \\
\hline Aglantha digitale (F. Müller, 1776) & S, SE, NE & $\begin{array}{l}\text { no specific } \\
\text { record }\end{array}$ & Ramirez \& Zamponi 1981 & $\mathrm{M}$ \\
\hline Aglaura hemistoma Péron \& Lesueur, 1810 & S, SE, NE & RS, SC, SP-PE & $\begin{array}{l}\text { Vannucci 1957a 1963, Moreira 1973, Goy } \\
\text { 1979, Navas-Pereira 1981 }\end{array}$ & $\mathrm{M}$ \\
\hline
\end{tabular}




\begin{tabular}{|c|c|c|c|c|}
\hline Homoeonema platygonon Browne, 1903 & $\mathrm{~S}, \mathrm{NE}$ & $\begin{array}{l}\text { no specific } \\
\text { record }\end{array}$ & Ramirez \& Zamponi 1981 & M \\
\hline Pantachogon haeckeli Maas, 1893 & $\mathrm{NE}$ & $\begin{array}{l}\text { no specific } \\
\text { record }\end{array}$ & Ramirez \& Zamponi 1981 & $\mathrm{M}$ \\
\hline Persa incolorata McCrady, 1859 & $\mathrm{~S}, \mathrm{NE}$ & $\begin{array}{l}\mathrm{RS} \text { (no specific } \\
\text { record for } \mathrm{NE} \\
\text { region) }\end{array}$ & $\begin{array}{l}\text { Thiel 1935, Navas-Pereira 1981, Ramirez } \\
\text { \& Zamponi } 1981\end{array}$ & $\mathrm{M}$ \\
\hline Rhopalonema velatum Gegenbaur, 1856 & S, SE, NE, N & $\begin{array}{l}\text { RS, SP, RJ, } \\
\text { BA, PA }\end{array}$ & $\begin{array}{l}\text { Vannucci 1957a, Goy 1979, Navas-Pereira } \\
19801981\end{array}$ & M \\
\hline Sminthea eurygaster Gegenbaur, 1856 & S, SE, NE & $\begin{array}{l}\text { RS (no specific } \\
\text { record for } \mathrm{SE} \\
\text { and } \mathrm{NE} \\
\text { regions) }\end{array}$ & $\begin{array}{l}\text { Navas-Pereira 1981, Ramirez \& Zamponi } \\
1981\end{array}$ & $\mathrm{M}$ \\
\hline \multicolumn{5}{|l|}{ Class Cubozoa } \\
\hline \multicolumn{5}{|l|}{ Order Cubomedusae } \\
\hline \multicolumn{5}{|l|}{ Family Carybdeidae } \\
\hline Tamoya haplonema F. Müller, 1859 & $\mathrm{~S}, \mathrm{SE}, \mathrm{NE}, \mathrm{N}$ & RS - AP & $\begin{array}{l}\text { F. Müller 1859b, Vannucci 1957b, } \\
\text { Morandini \& Marques 1997, Mianzan \& } \\
\text { Cornelius } 1999\end{array}$ & $\mathrm{M}$ \\
\hline Tripedalia cystophora Conant, 1897 & $\mathrm{~N}$ & PA & $\begin{array}{l}\text { Morandini \& Marques 1997, Mianzan \& } \\
\text { Cornelius } 1999\end{array}$ & $\mathrm{M}$ \\
\hline \multicolumn{5}{|l|}{ Family Chirodropidae } \\
\hline Chiropsalmus quadrumanus (F. Müller, 1859) & S, SE, NE, N & $\mathrm{SC}-\mathrm{AP}$ & $\begin{array}{l}\text { F. Müller 1859b, Vannucci 1954, 1957b, } \\
\text { Morandini \& Marques 1997, Marques et } \\
\text { al. 1997, Mianzan \& Cornelius } 1999\end{array}$ & $\mathrm{M}$ \\
\hline \multicolumn{5}{|l|}{ Class Scyphozoa } \\
\hline \multicolumn{5}{|l|}{ Order Coronatae } \\
\hline \multicolumn{5}{|l|}{ Family Atollidae } \\
\hline Atolla chuni Vanhöffen, 1902 & S, SE, NE, N & $\begin{array}{l}\mathrm{RS}-\mathrm{AP} \\
\text { (offshore) }\end{array}$ & Mianzan \& Cornelius 1999 & $\mathrm{M}$ \\
\hline Atolla wyvillei Haeckel, 1880 & S, SE, NE, N & $\begin{array}{l}\text { RS - AP } \\
\text { (offshore) }\end{array}$ & Mianzan \& Cornelius 1999 & $\mathrm{M}$ \\
\hline \multicolumn{5}{|l|}{ Family Linuchidae } \\
\hline Linuche unguiculata (Swartz, 1788) & SE & SP & $\begin{array}{l}\text { Silveira \& Morandini 1998a 1998b, } \\
\text { Haddad Jr. et al. } 2001\end{array}$ & $\mathrm{P} / \mathrm{M}$ \\
\hline \multicolumn{5}{|l|}{ Family Nausithoidae } \\
\hline Nausithoe aurea Silveira \& Morandini 1997 & SE, NE & SP, BA & $\begin{array}{l}\text { Silveira \& Morandini 1997, Mianzan \& } \\
\text { Cornelius 1999, Morandini \& Silveira } \\
\text { 2001a 2001b }\end{array}$ & $\mathrm{P} / \mathrm{M}$ \\
\hline Nausithoe punctata Kölliker, 1853 & S, SE, NE, N & $\begin{array}{l}\text { RS - AP } \\
\text { (offshore) }\end{array}$ & $\begin{array}{l}\text { Vanhöffen 1908, Alvariño 1968, Goy } \\
\text { 1979, Mianzan \& Cornelius } 1999\end{array}$ & M \\
\hline Nausithoe sp. & $\mathrm{S}$ & $\mathrm{RS}$ & Horta et al. 2001 & $\mathrm{P}$ \\
\hline $\begin{array}{l}\text { Stephanoscyphistoma corniformis (Komai, } \\
\text { 1936) }\end{array}$ & SE & $\mathrm{SP}, \mathrm{RJ}$ & Silveira \& Morandini 1996 & $\mathrm{P}$ \\
\hline $\begin{array}{l}\text { Stephanoscyphistoma simplex (Kirkpatrick, } \\
\text { 1890) }\end{array}$ & $\mathrm{NE}$ & $\begin{array}{l}\text { CE (ca. } 750 \mathrm{~km} \\
\text { offshore) }\end{array}$ & Kramp 1951 & $\mathrm{P}$ \\
\hline \multicolumn{5}{|l|}{ Family Periphyllidae } \\
\hline Periphylla periphylla (Péron \& Lesueur, 1809) & S, SE, NE, N & $\begin{array}{l}\text { RS - AP } \\
\text { (offshore) }\end{array}$ & Mianzan \& Cornelius 1999 & $\mathrm{M}$ \\
\hline \multicolumn{5}{|l|}{ Order Rhizostomeae } \\
\hline \multicolumn{5}{|l|}{ Family Cassiopeidae } \\
\hline Cassiopea xamachana R.P. Bigelow, 1892 & SE & SP & Silveira et al. 2000 & $\mathrm{P} / \mathrm{M}$ \\
\hline
\end{tabular}




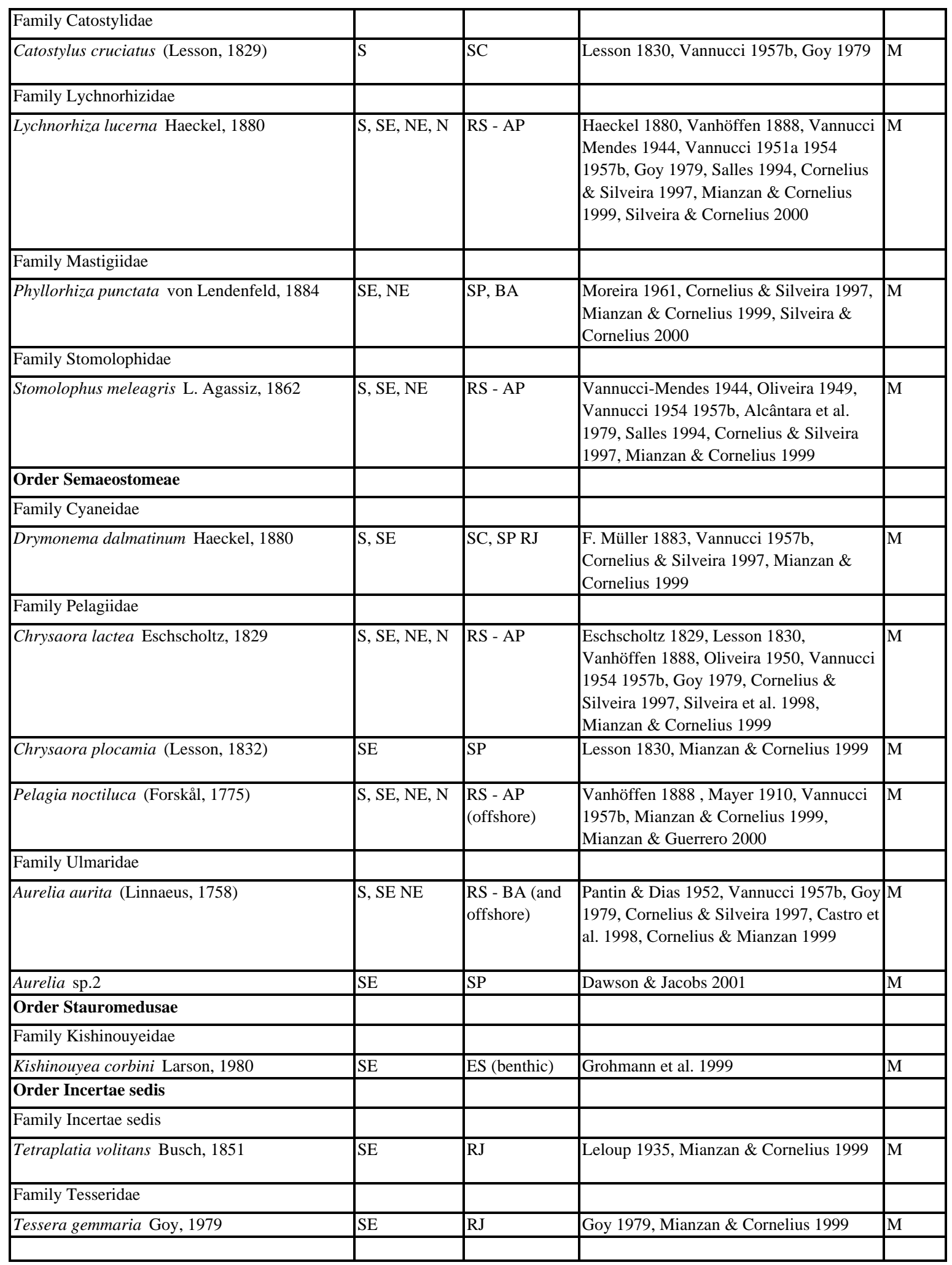



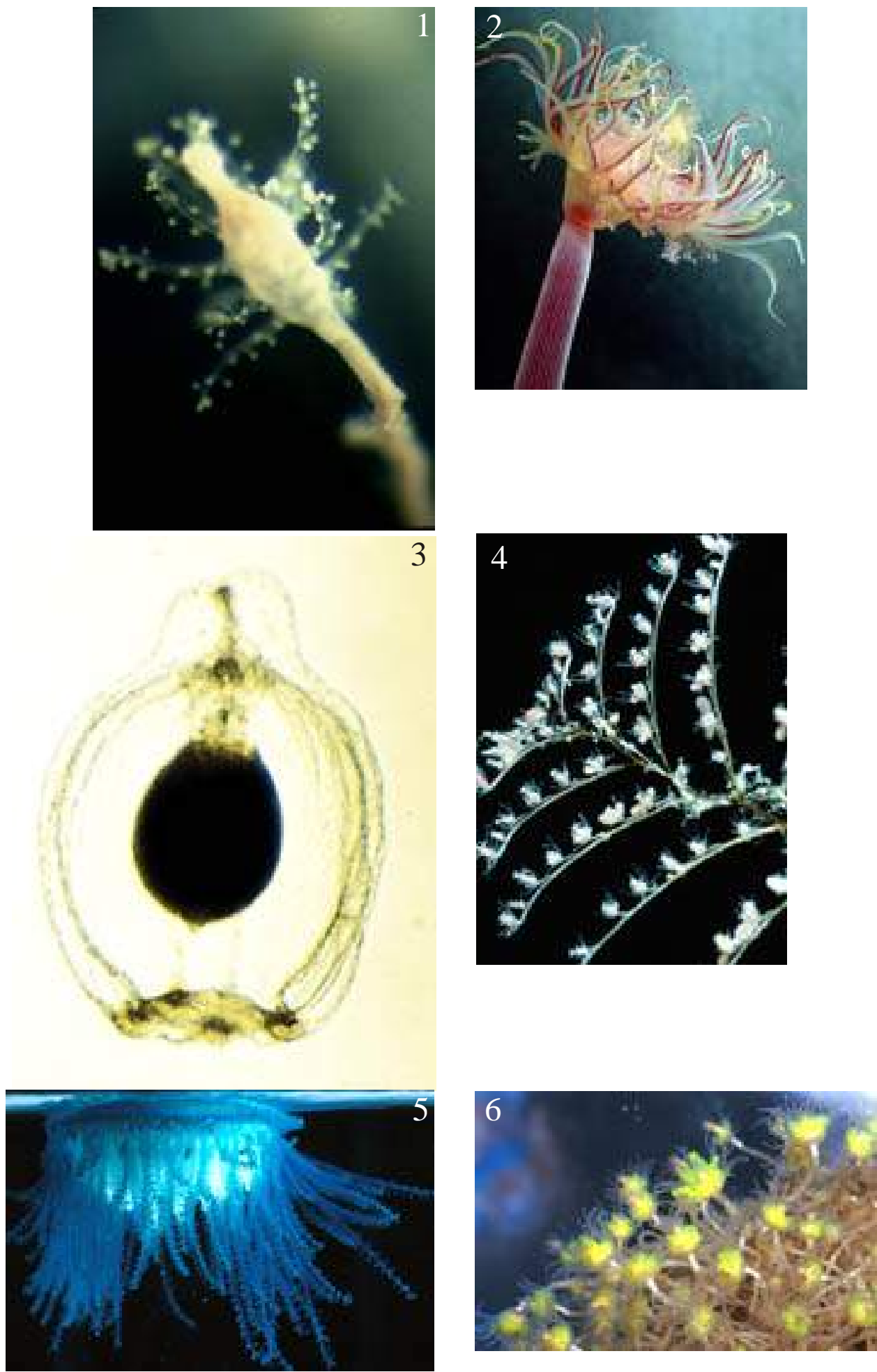

Figure 1. Asyncoryne ryniensis Warren, 1908 polyp photo Alvaro E. Migotto

Figure 2. Corymorpha januarii Steenstrup, 1854 polyp photo Alvaro E. Migotto

Figure 3. Corymorpha januarii Steenstrup, 1854 medusa photo Alvaro E. Migotto

Figure 4. Pennaria disticha Goldfuss, 1820 colony photo Alvaro E. Migotto

Figure 5. Porpita umbella F. Müller, 1776 colony photo Alvaro E. Migotto

Figure 6. Ectopleura obypa Migotto \& Marques, 1999 colony photo Alvaro E. Migotto 

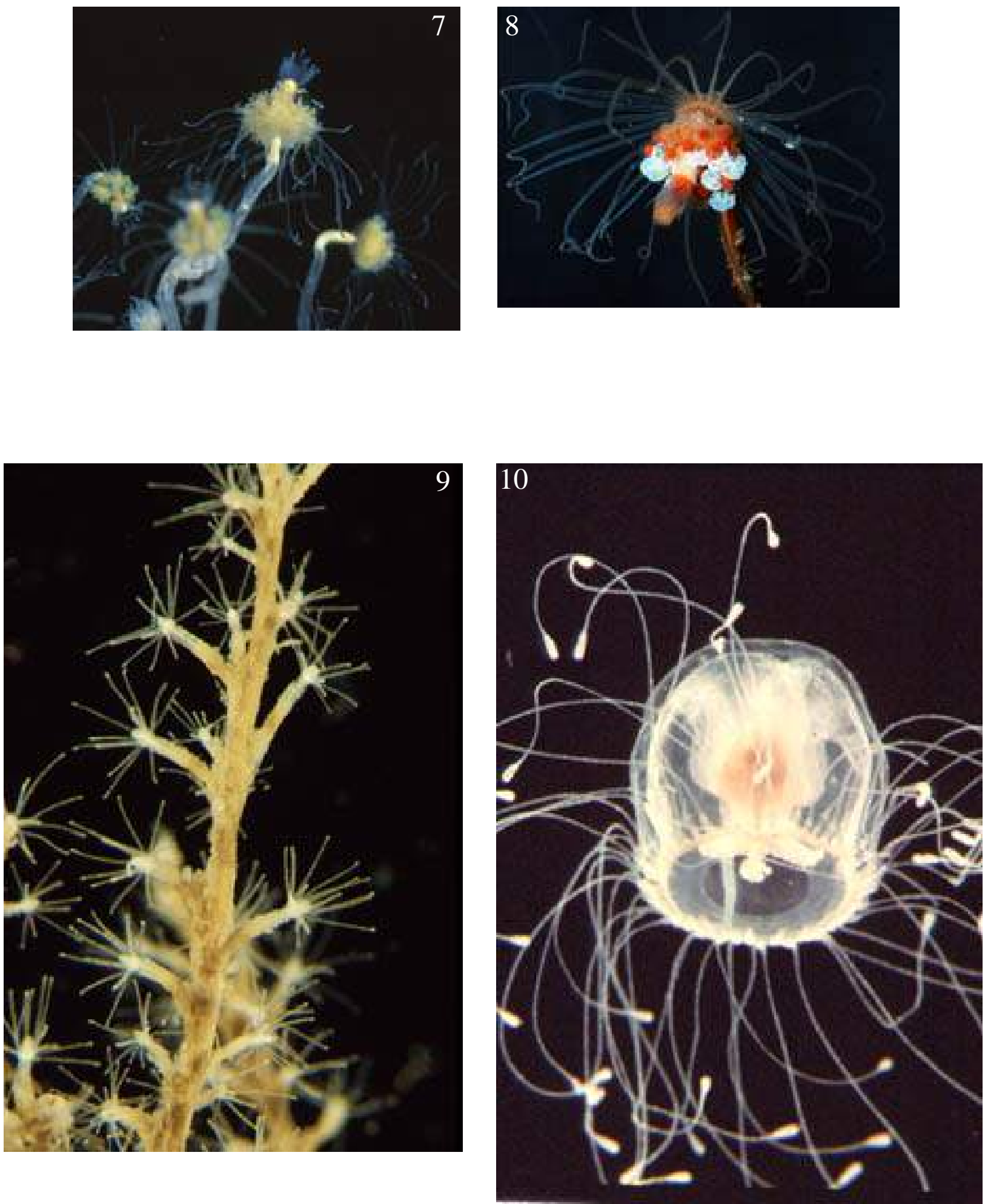

Figure 7. Ectopleura obypa Migotto \& Marques, 1999 polyp photo Alvaro E. Migotto

Figure 8. Ralpharia sanctisebastiani (Silveira \& Migotto, 1984) polyp photo Alvaro E. Migotto

Figure 9. Bougainvillia rugosa Clarke, 1882 colony photo Alvaro E. Migotto

Figure 10. Turritopsis nutricula McCrady, 1859 medusa photo Alvaro E. Migotto 

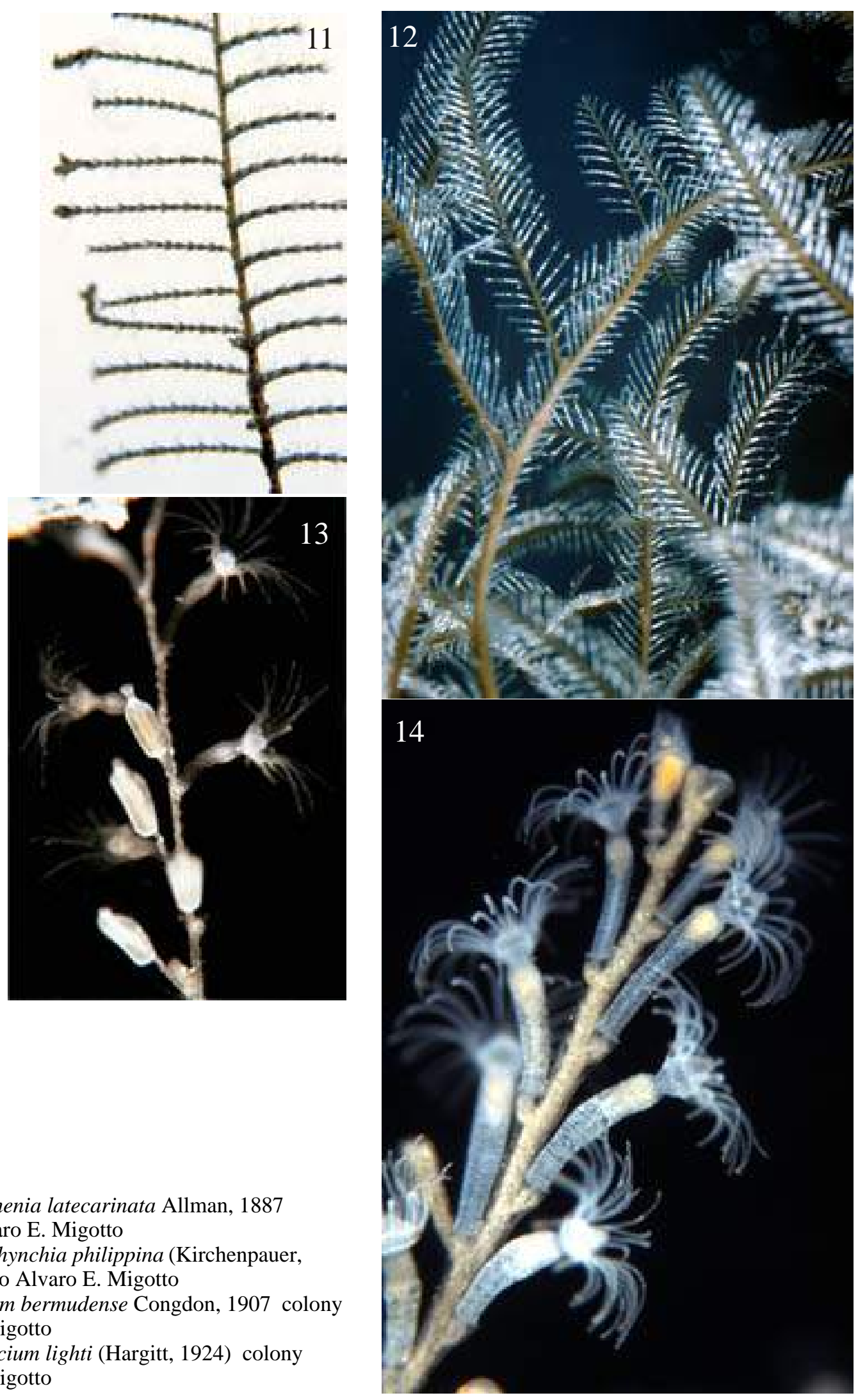

Figure 11. Aglaophenia latecarinata Allman, 1887 colony photo Alvaro E. Migotto

Figure 12. Macrorhynchia philippina (Kirchenpauer, 1872 colony photo Alvaro E. Migotto

Figure 13. Halecium bermudense Congdon, 1907 colony photo Alvaro E. Migotto

Figure 14. Nemalecium lighti (Hargitt, 1924) colony photo Alvaro E. Migotto 

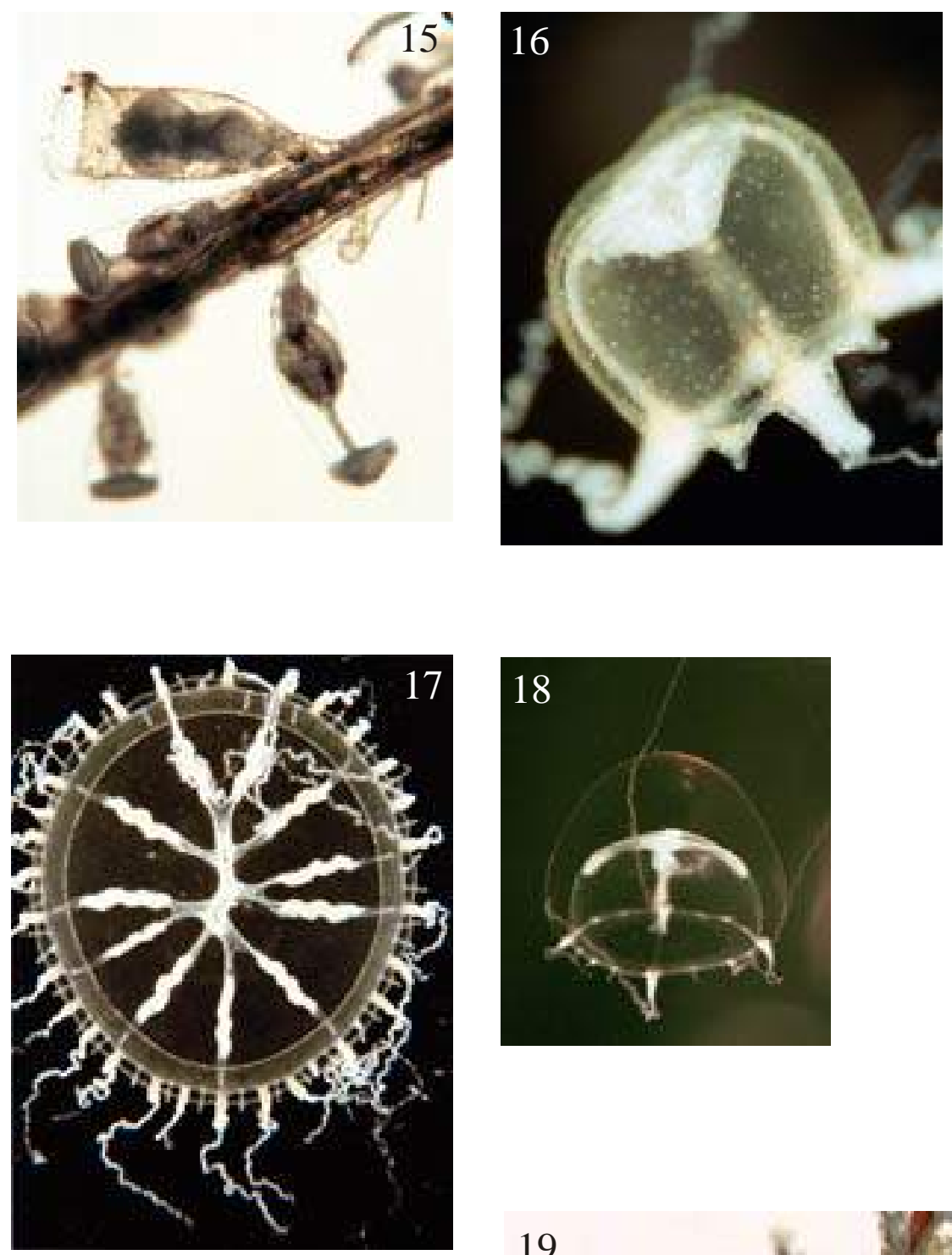

Figure 15. Hebella furax Millard, 1957 colony photo Alvaro E. Migotto

Figure 16. Hebella furax Millard, 1957 newly released medusa photo Alvaro E. Migotto

Figure 17. Hebella furax Millard, 1957 adult medusa photo Alvaro E. Migotto

Figure 18. Hebella scandens (Bale, 1888 medusa photo Alvaro E. Migotto

Figure 19. Dentitheca bidentata Jäderholm, 1920 colony photo Alvaro E. Migotto

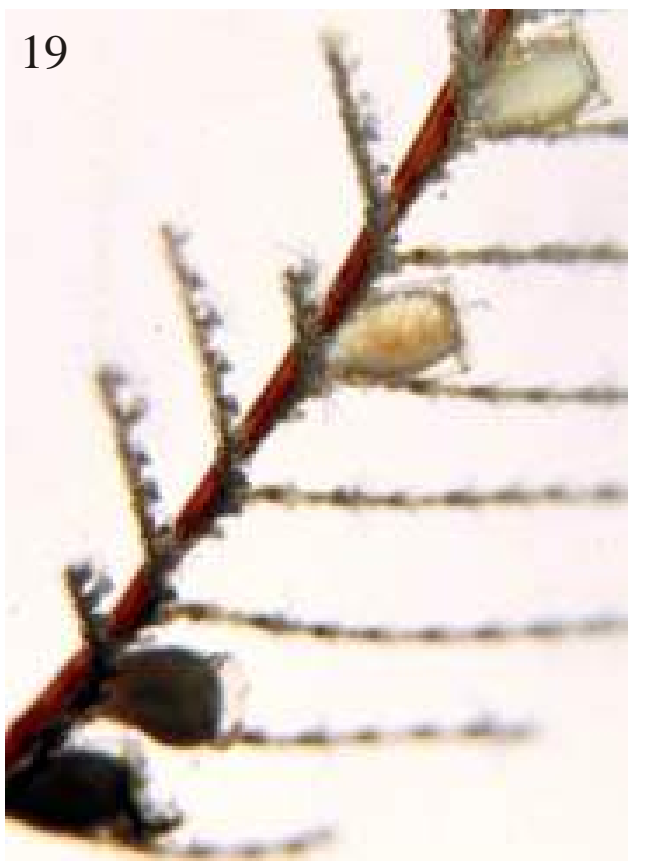

http://www.biotaneotropica.org.br 

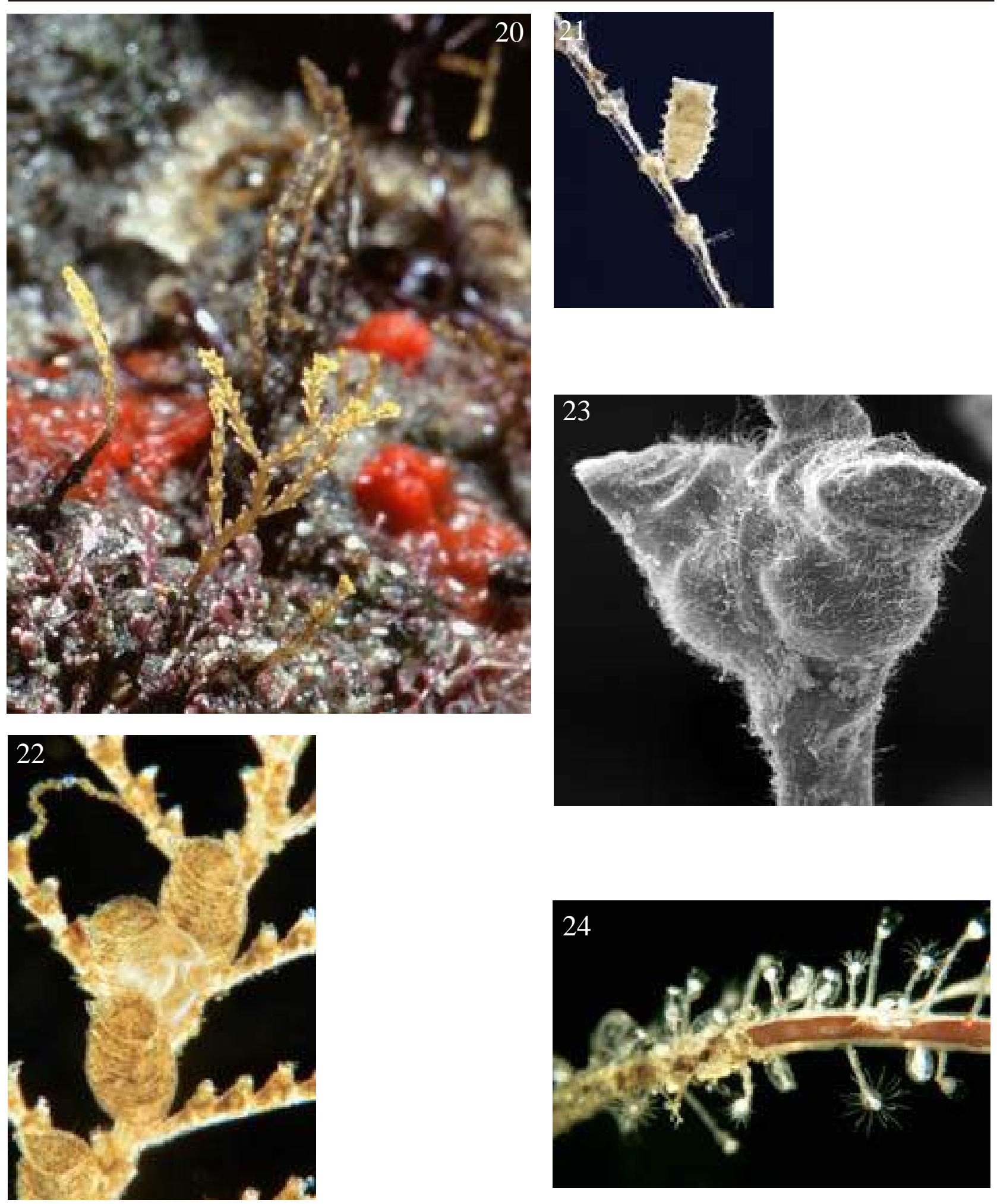

Figure 20. Dynamena crisioides Lamouroux, 1824 colony photo Alvaro E. Migotto Figure 21. Sertularia loculosa Busk, 1852 colony photo Alvaro E. Migotto

Figure 22. Sertularia marginata Kirchenpauer, 1864 colony photo Alvaro E. Migotto Figure 23. Sertularia vervoorti Migotto \& Calder, 1998 photo Alvaro E. Migotto Figure 24. Clytia cf. gracilis sp. 1 colony photo Alberto Lindner 

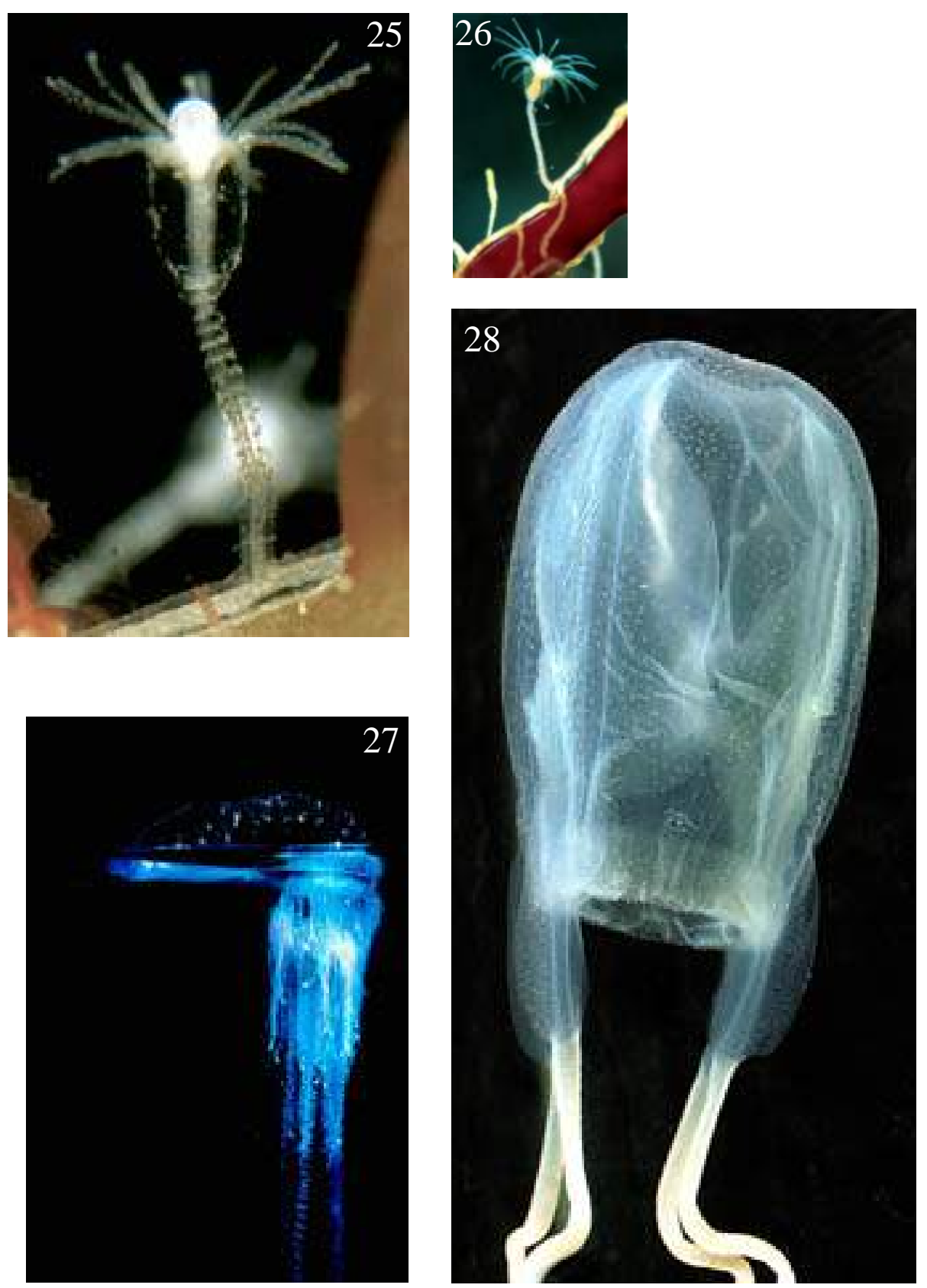

Figure 25. Clytia $c f$. gracilis sp. 1 hydranth photo Alberto Lindner Figure 26. Clytia noliformis auct. hydranth photo Alberto Lindner

Figure 27. Physalia physalis Linnaeus 1758 colony photo Alvaro E. Migotto

Figure 28. Tamoya haplonema F. Müller, 1859 medusa photo Alvaro E. Migotto 

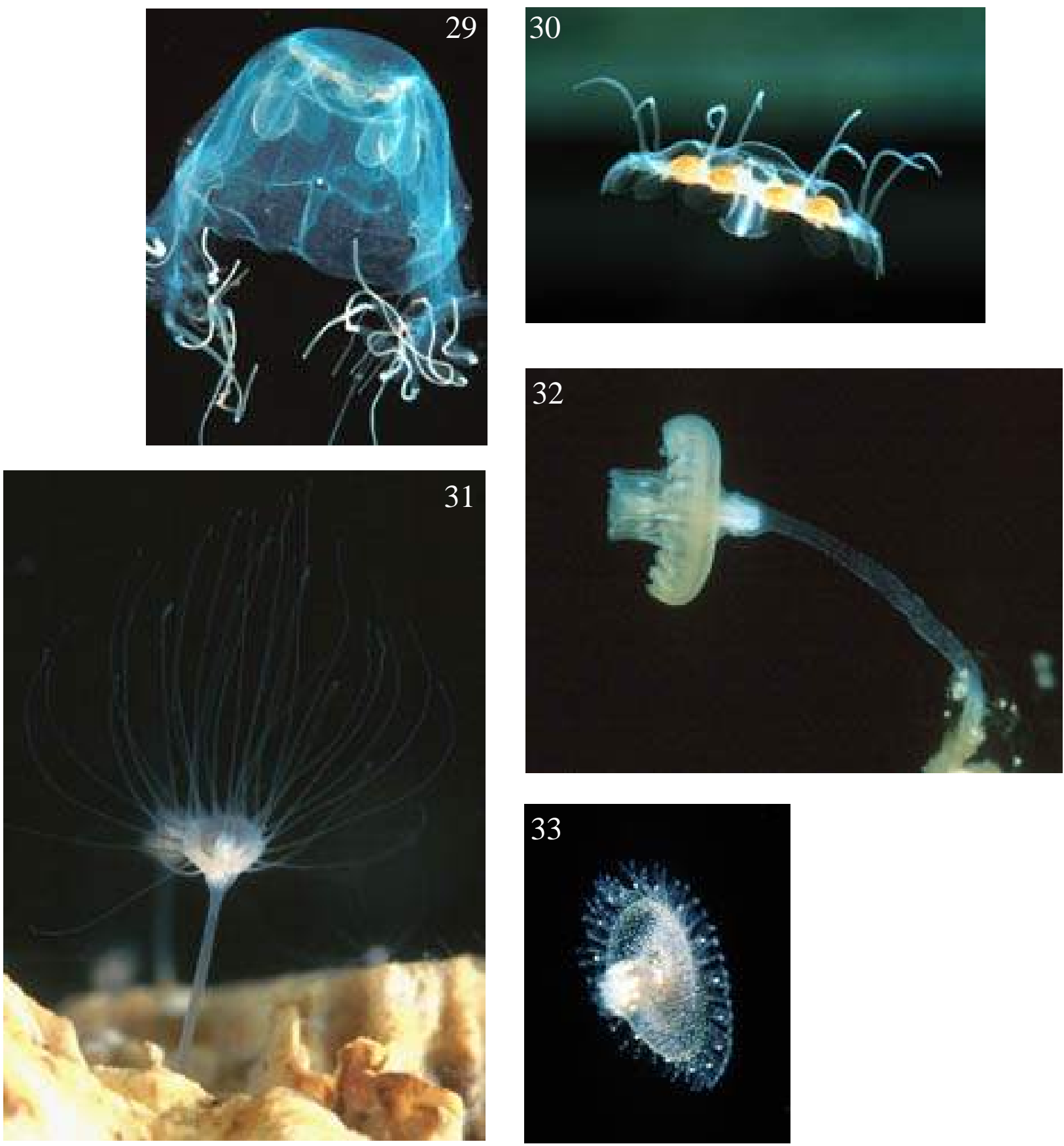

Figure 29. Chiropsalmus quadrumanus (F. Müller, 1859) medusa photo Alvaro E. Migotto Figure 30. Nausithoe aurea medusa photo Alvaro E. Migotto

Figure 31. Cassiopea xamachana R.P. Bigelow, 1892 scyphistoma photo Alvaro E. Migotto Figure 32. Cassiopea xamachana R.P. Bigelow, 1892 strobila photo Alvaro E. Migotto Figure 33. Cassiopea xamachana R.P. Bigelow, 1892 ephyra photo Alvaro E. Migotto 

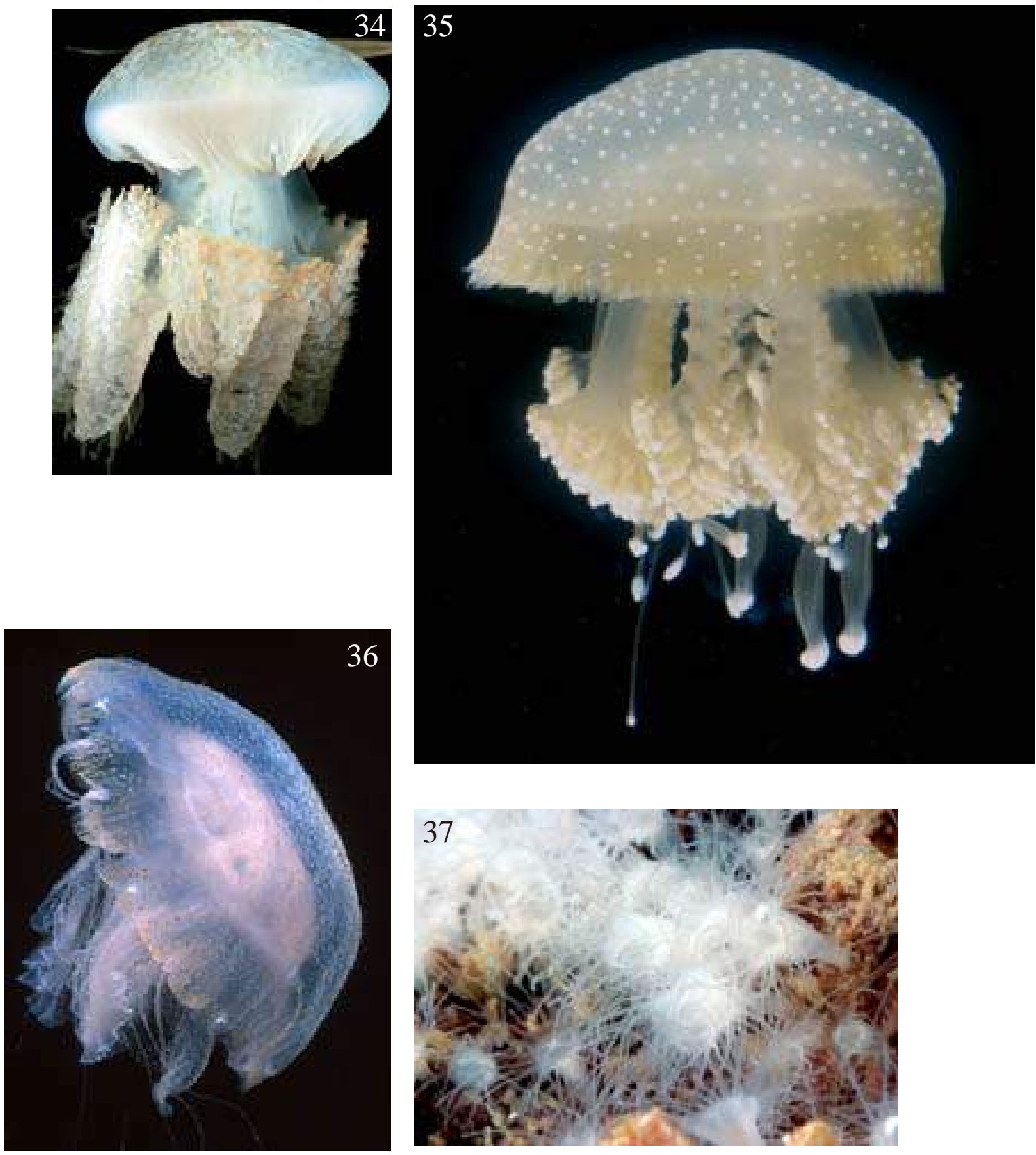

Figure 34. Lychnorhiza lucerna Haeckel, 1880 medusa photo Alvaro E. Migotto

Figure 35. Phyllorhiza punctata von Lendenfeld, 1884 medusa photo Fábio L. da Silveira

Figure 36. Chrysaora lactea Eschscholtz, 1829 medusa photo Alvaro E. Migotto

Figure 37. Aurelia aurita (Linnaeus, 1758) scyphistoma photo Alvaro E. Migotto 